\title{
Deflection and deceleration of hydrogen Rydberg molecules in inhomogeneous electric fields
}

\author{
Y. Yamakita, ${ }^{\text {a) }}$ S. R. Procter, A. L. Goodgame, and T. P. Softley ${ }^{\text {b) }}$ \\ Department of Chemistry, University of Oxford, Chemistry Research Laboratory, Mansfield Road, \\ Oxford OX1 3TA, United Kingdom \\ F. Merkt \\ Laboratorium für Physikalische Chemie, ETH, Zurich CH-8093, Switzerland
}

(Received 23 March 2004; accepted 26 April 2004)

\begin{abstract}
Hydrogen molecules are excited in a molecular beam to Rydberg states around $n=17-18$ and are exposed to the inhomogeneous electric field of an electric dipole. The large dipole moment produced in the selected Stark eigenstates leads to strong forces on the $\mathrm{H}_{2}$ molecules in the inhomogeneous electric field. The trajectories of the molecules are monitored using ion-imaging and time of flight measurements. With the dipole rods mounted parallel to the beam direction, the high-field-seeking and low-field-seeking Stark states are deflected towards and away from the dipole, respectively. The magnitude of the deflection is measured as a function of the parabolic quantum number $k$ and of the duration of the applied field. It is also shown that a large deflection is observed when populating the $(17 d 2)_{1}$ state at zero field and switching the dipole field on after a delay. With the dipole mounted perpendicular to the beam direction, the molecules are either accelerated or decelerated as they move towards the dipole. The Rydberg states are found to survive for over $100 \mu$ s after the dipole field is switched off before being ionized at the detector and the time of flight is measured. A greater percentage change in kinetic energy is achieved by initial seeding of the beam in helium or neon followed by inhomogeneous field deceleration/acceleration. Molecular dynamics trajectory simulations are presented highlighting the extent to which the trajectories can be predicted based on the known Stark map. The spectroscopy of the populated states is discussed in detail and it is established that the $N^{+}=2, J=1, M_{J}=0$ states populated here have a special stability with respect to decay by predissociation. (c) 2004 American Institute of Physics. [DOI: 10.1063/1.1763146]
\end{abstract}

\section{INTRODUCTION}

In this paper we report on the control of molecular translational motion by using the force created by an inhomogeneous electric field on the Rydberg states of the $\mathrm{H}_{2}$ molecule. Controlling the translational motion of gas-phase molecules, particularly through the use of supersonic molecular beams, has played an important role in the development of experiments in reaction dynamics and spectroscopy. ${ }^{1}$ Narrowing the velocity distribution to the order of $1 \mathrm{~K}$ makes possible scattering experiments with well-defined collision energy (both in the gas phase and at the gas-solid interface) and also spectroscopic measurements with reduced Doppler contribution to the linewidths. Interest in the methodology of translational control has been increased by the recent efforts to create ultracold molecules, following the successful development of laser cooling methods for atoms. ${ }^{2-5} \mathrm{~A}$ gas sample is commonly defined as "ultracold" if it shows a velocity distribution characteristic of a temperature less than $1 \mathrm{mK}$. The main approaches employed have been: (a) the use of helium buffer-gas cooling combined with magnetic trapping, demonstrated for $\mathrm{CaH}$ to give temperatures around 240

\footnotetext{
a) Present address: Department of Chemistry, Graduate School of Science, Tohoku University, Sendai 980-8578, Japan.

${ }^{b}$ Author to whom correspondence should be addressed. Electronic mail: tim.softley@chem.ox.ac.uk
}

$\mathrm{mK} ; ;^{7}$ (b) the use of inhomogeneous electric fields to decelerate molecular beams of dipolar molecules, ${ }^{8-10}$ for which temperatures of around $10 \mathrm{mK}$ have been achieved for $\mathrm{ND}_{3}$; (c) the photoassociation of alkali-metal atoms cooled in magneto-optical traps ${ }^{11,12}$ to make alkali-metal dimers, giving temperatures as low as $20 \mu \mathrm{K}$. Very recently Ketterle and co-workers have observed Bose-Einstein condensation by evaporative cooling of ${ }^{6} \mathrm{Li}$ atoms in an optical dipole trap near a Feshbach resonance. ${ }^{13}$

Method (b), developed by Meijer and co-workers (see Ref. 10 for a recent review) and known as the "Stark decelerator" approach, is perhaps the most general, being applicable in principle to any molecule having a significant dipole moment and showing a first-order Stark effect. It is an extension of several decades of development of the use of inhomogeneous fields provided by multipolar devices to deflect, focus and orient beams of dipolar molecules. ${ }^{14-16}$ The principle employed is that the energy of a given eigenstate in an inhomogeneous field varies with spatial position, and therefore a force $(\mathbf{F})$ is exerted in the direction of decreasing energy $(\mathbf{F}=-\boldsymbol{\nabla E})$. The eigenstates can be classified as either high-field seeking or low-field seeking according to whether the energy decreases or increases with increasing field, respectively. To decelerate a molecule in a low-field seeking state, it is necessary to arrange that the field increases in the forward direction of motion so that the molecule will lose an 
amount of kinetic energy equal to the energy increase of the state in the field. The Stark shift of a molecule in its electronic ground state is normally very small-for $\mathrm{ND}_{3}$ the $J$ $=1, M_{J} K=-1$ level changes in energy by only $\approx 1.7 \mathrm{~cm}^{-1}$ for a field rise of $150 \mathrm{kV} / \mathrm{cm},{ }^{10}$ which represents a small change of kinetic energy compared to the typical kinetic energy of molecules in a molecular beam $\left(10-1000 \mathrm{~cm}^{-1}\right)$. Meijer and co-workers have shown that by using a sequence of 63 high-voltage deceleration stages, $\mathrm{ND}_{3}$ molecules can be decelerated to very low mean velocities and trapped at an effective temperature of $25 \mathrm{mK} .^{10}$

As we have reported previously, ${ }^{17-20}$ and as discussed by others, ${ }^{21,22}$ the Rydberg states of atoms and molecules generally show a very large first-order Stark shift, which is equivalent to the molecule possessing a large permanent dipole moment in the excited state, irrespective of whether it has a ground-state dipole moment. It should therefore be possible to decelerate Rydberg molecules using a much lower field inhomogeneity and the methodology should even be applicable to atoms and molecules with no permanent dipole moment. The extreme components of the $n=17$ Stark manifold for $\mathrm{H}_{2}$ show energy versus field gradients that are approximately 1000 times greater than for the ground state of $\mathrm{ND}_{3}$ and hence a dipole moment that is 1000 times greater. It is therefore of importance to investigate whether this advantage of using Rydberg excitation for Stark deceleration outweighs the two main disadvantages: first, these states are expected to have short lifetimes with respect to decay processes such as predissociation, autoionization, or spontaneous emission; second, there is a requirement to produce these states with sufficient number density by spectroscopic selection using vacuum ultraviolet or multiphoton excitation. Indeed at the outset of the present experiments it was not clear whether the lifetimes of the $\mathrm{H}_{2}$ Rydberg states would be sufficient to achieve any translational control at all. A key issue that is re-emphasized in the work presented here, and must be considered in the design of experiments of this type, is that the fields employed for controlling the motion of Rydberg molecules also have a significant effect on the lifetimes.

In a recent paper $^{19}$ we presented a preliminary report showing that $\mathrm{H}_{2}$ molecules excited to Rydberg states of principal quantum number $n=17$ are sufficiently long lived to demonstrate for the first time the controlled deflection of a neutral nonpolar nonmagnetic molecule in a static electric field. We went on to demonstrate that a beam of $\mathrm{H}_{2}$ molecules could be slowed down or accelerated by the application of inhomogeneous electric fields, pointing to the possibility of trapping and cooling such molecules. In this paper we present a full description of these measurements and a broader range of results. The underlying spectroscopy is discussed in detail and results of Monte Carlo simulations are presented to support our conclusions.

\section{EXPERIMENT}

\section{A. Excitation scheme}

A population of $\mathrm{H}_{2}$ Rydberg states is produced in a pulsed, skimmed molecular beam using a vacuum ultraviolet
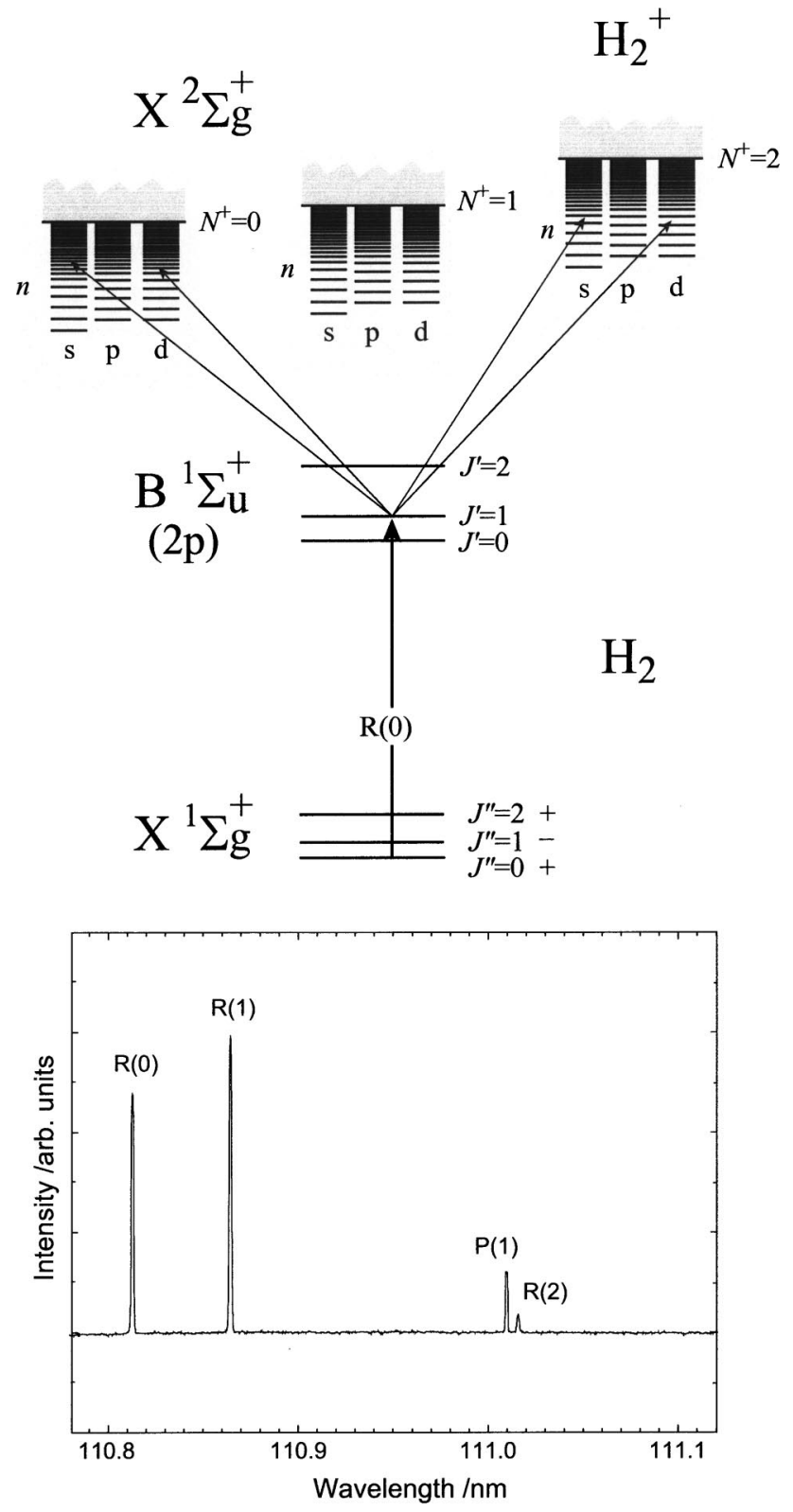

FIG. 1. (a) VUV+UV doubly resonant two-photon excitation scheme to excite $n s / n d$ Rydberg states of $\mathrm{H}_{2}$. (b) $\left(1+1^{\prime}\right)$ resonantly enhanced multiphoton ionization spectrum of the $B \leftarrow X(0,0)$ band of $\mathrm{H}_{2}$.

(VUV)-UV doubly resonant excitation scheme illustrated in Fig. 1(a). The VUV frequency is resonant with the $R(0) B{ }^{1} \Sigma_{u}^{+}\left(\nu^{\prime}=0\right)-X^{1} \Sigma_{g}^{+}\left(\nu^{\prime \prime}=0\right)$ one-photon transition of para-hydrogen at an excitation energy of $90242.33 \mathrm{~cm}^{-1}$ (Ref. 23) populating only the $J^{\prime}=1$ level in the $B{ }^{1} \Sigma_{u}^{+}\left(\nu^{\prime}\right.$ $=0)$ intermediate state. The very simple spectrum of the $B-X(0-0)$ band in a neat- $\mathrm{H}_{2}$ molecular beam is shown in Fig. 1(b). The UV frequency (laser 2) is resonant with the one-photon transitions from the intermediate state to the Rydberg series converging on the ionization thresholds associated with the para- $\mathrm{H}_{2}$ vibration-rotation levels, $\nu^{+}=0$, $N^{+}=0$ and 2 of the $X^{2} \Sigma_{g}^{+}$ground ionic state at a total energy of approximately $124000 \mathrm{~cm}^{-1}$ above the ground state $\left(I E=124417.507(10) \mathrm{cm}^{-1}\right) .{ }^{24}$ The final states of the transitions from the ungerade intermediate state must have gerade symmetry overall, and as the ion core of the Rydberg 


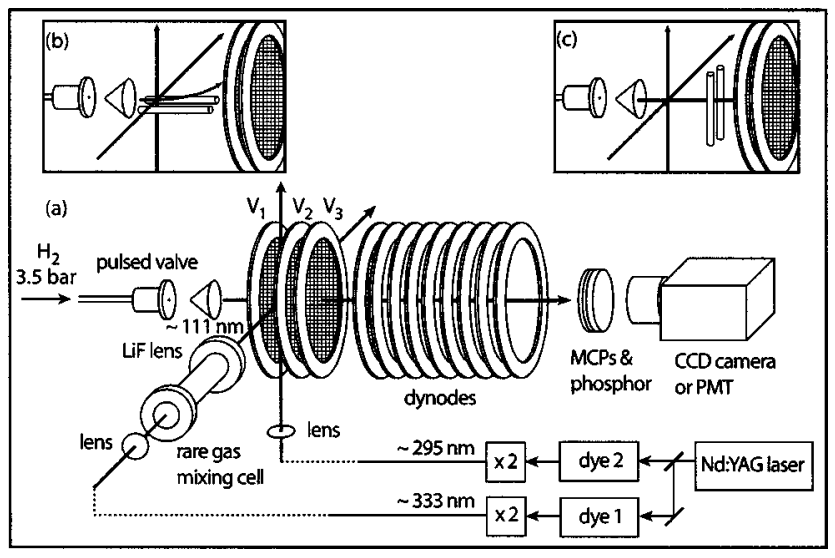

FIG. 2. Experimental apparatus. (a) The configuration shown is used to excite the $\mathrm{H}_{2}$ molecules to Rydberg states in a homogeneous field between grids $V_{1}$ and $V_{2}$ and then field ionize the molecules between $V_{2}$ and $V_{3}$. (b) For deflection experiments, excitation occurs in the inhomogeneous field of an electric dipole mounted parallel to the molecular beam direction. (c) For deceleration/acceleration, the apparatus is as in (b) except that the dipole rods are oriented perpendicular to the molecular beam direction.

states is of ${ }^{2} \Sigma_{g}^{+}$electronic symmetry, it is only the even- $\ell$ Rydberg series, primarily $n s$ and $n d$ states, that are accessible in the UV excitation step. Furthermore the $\Delta J=0, \pm 1$ selection rule restricts excitation to states with total angular momentum $J=0,1$, or 2 , where $J$ is in this case the vector sum of the core rotation $N^{+}$and the Rydberg electron angular momentum $\ell$, assuming a resultant electron spin of zero. Thus, the main series accessible from a $J^{\prime}=1$ intermediate state [notated $\left.\left(n \ell N^{+}\right)_{J}\right]$ are $(n s 0)_{0},(n s 2)_{2},(n d 0)_{2}$, $(n d 2)_{0},(n d 2)_{1}$, and $(n d 2)_{2}$ although as shown below, only a subset of these are accessible for a specific choice of laser polarizations. The $(n d 4)_{2}$ series is also accessible in principle, although it might be expected that the transition probability for this "T-branch" process $\left(N^{+}-J^{\prime}=3\right)$ would be very low.

An overview of the experimental apparatus is shown in Fig. 2. The VUV beam $(\lambda=110.81 \mathrm{~nm})$, which intersects the molecular beam perpendicularly and is focused using a lithium fluoride lens $(f=70 \mathrm{~mm})$, is obtained by nonresonant frequency tripling of the frequency-doubled output (10 $\mathrm{Hz}, 6 \mathrm{~ns}, 5 \mathrm{~mJ} /$ pulse) of a Nd:YAG pumped dye laser (Quanta Ray PDL3) in a $20 \mathrm{~cm}$ long cylindrical cell containing a $\mathrm{Kr} / \mathrm{Ar}$ mixture (1180 mbar/200 mbar). The relative efficiency of the tripling process for different gas mixtures as a function of laser wavelength is illustrated in Fig. 3. The UV beam ( $\lambda=291-296 \mathrm{~nm}, 5 \mathrm{~mJ} /$ pulse) is provided by a second frequency-doubled dye laser (Quanta Ray PDL3) pumped by the same Nd:YAG laser (Spectra Physics GCR 290), and is weakly focused using a $f=285 \mathrm{~mm}$ lens; its direction is perpendicular to both the molecular beam and the VUV beam. The polarizations of laser 1 and laser 2 are set to be mutually perpendicular, and consequently the overall selection rule applicable to the two-step excitation is $\Delta M_{J}= \pm 1$, defining the quantization axis with respect to one of these polarizations. This implies, given the initial state has $J^{\prime \prime}=0$ and $M_{J}^{\prime \prime}=0$, that the final states of the double-resonant excitation must have $M_{J}= \pm 1$ and so $J$ (the final-state total angular momentum) must be greater than 0 , i.e., the zero-field transition

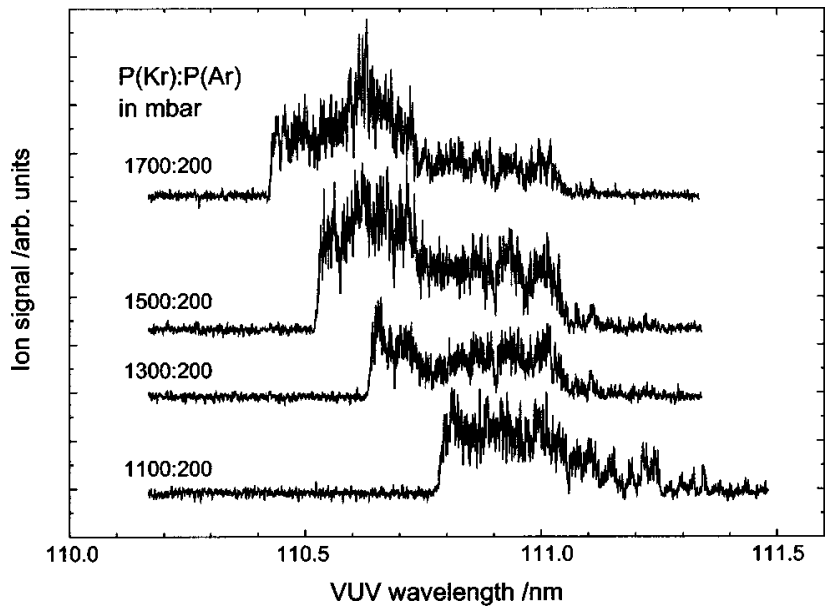

FIG. 3. Third harmonic generation efficiency in the $110-111.5 \mathrm{~nm}$ region as a function of $\mathrm{Kr} / \mathrm{Ar}$ gas mix and wavelength.

moments to the $(n s 0)_{0}$ and $(n d 2)_{0}$ states must be zero; this conclusion holds irrespective of the arbitrary choice of quantization axis.

\section{B. Electric field configurations and detection}

\section{Spectroscopic measurements using homogeneous fields}

In order to determine the Stark map showing the variation of the Rydberg energy levels with electric field, as described in Sec. III B, a set of three parallel grid electrodes (90\% transmitting Ni mesh with 45 wires per inch) of $8 \mathrm{~cm}$ diameter are mounted in the excitation region to provide two separate homogeneous field regions [see Fig. 2(a)]. Grids 1 and 2 are spaced by $1.5 \mathrm{~cm}$, whereas grids 2 and 3 are spaced by $1.0 \mathrm{~cm}$. For these measurements the two laser polarizations are both perpendicular to the field direction and also perpendicular to each other. The excitation point is $7.5 \mathrm{~cm}$ from the molecular beam skimmer and $11.4 \mathrm{~cm}$ from the 0.5 $\mathrm{mm}$ orifice nozzle, and is midway between grids 1 and 2 . The molecules are excited in the presence of a variable homogeneous field $(0-1000 \mathrm{~V} / \mathrm{cm})$ applied between grids 1 and 2 and then they pass into the region between grids 2 and 3 where they are ionized by a pulsed homogeneous field of magnitude $\approx 5000 \mathrm{~V} / \mathrm{cm}$. In all these spectroscopic experiments, neat $\mathrm{H}_{2}$ gas is used (backing pressure 3.5 bar) resulting in a beam that travels at a velocity of $2600 \mathrm{~ms}^{-1}$. The transit time from the excitation region into the center of the field ionization zone is typically of the order of $3.2 \mu \mathrm{s}$. The pulsed ionizing field accelerates the resultant molecular ions into a field-free flight tube and the ions are detected by a position-sensitive detector, comprising a microchannel plates (MCPs)/phosphor screen assembly imaged by an ISIS 3000 gated inductively charge coupled device (ICCD) camera (Photonic Science, $760 \times 576$ pixels), or viewed by a photomultiplier tube (PMT) for recording the total ion signal.

\section{Deflection experiments}

For the deflection experiments (results reported in Sec. III C), grid 1 is removed and the homogeneous field is replaced by an inhomogeneous field provided by a pair of pol- 

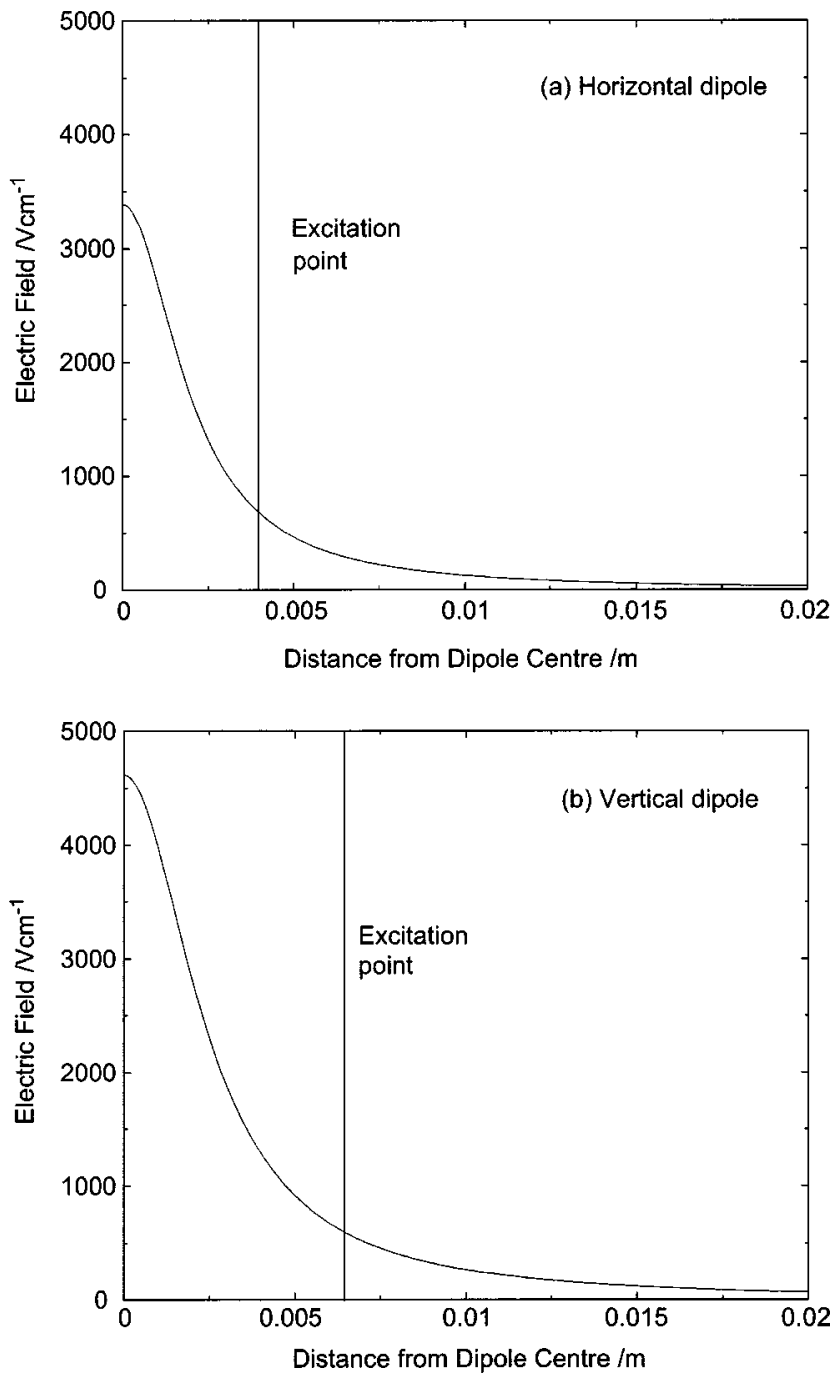

FIG. 4. (a) Calculated fields for the dipole used in the deflection experiments [Fig. 2(b)] along a vertical line that passes through the center of the gap between the dipole rods. (b) Calculated fields for the dipole used in the deceleration experiments [Fig. 2(c)] as a function of position along the molecular beam axis.

ished nickel cylindrical rods, which act as an electric dipole. The dipole rods are parallel to one another and oriented parallel to the horizontal molecular beam direction as shown in Fig. 2(b). The molecular beam passes $3.95 \mathrm{~mm}$ above the center of the pair of rods (of length $8 \mathrm{~cm}$, diameter $0.9 \mathrm{~mm}$, and center-to-center separation $4 \mathrm{~mm}$ ) that are charged by a pulsed potential of up to $\pm 700 \mathrm{~V}$. The molecules are excited at a position $2 \mathrm{~cm}$ before the end of the dipole. Along the central axis of the molecular beam, the electric field of the dipole is directed horizontally and is perpendicular to the molecular beam direction. The field decreases in magnitude in the vertical direction as one moves away from the dipole as illustrated in Fig. 4(a). The Rydberg molecules travel up to $18 \mathrm{~mm}$ in the field of the electric dipole, which is then switched off at a selected time up to $7 \mu$ s after excitation.

The molecules then pass into the region between grids 2 and 3 where a pulsed homogeneous field of up to $5 \mathrm{kV} / \mathrm{cm}$ can be applied to ionize the Rydberg states and accelerate them over a distance of $26.7 \mathrm{~cm}$ from the center of the fieldionization region to the position-sensitive detector through the grounded dynode stack and flight tube. A mesh grid is mounted at the front end of the dynode stack. The ions arrive at the detector 10-11 $\mu$ s after the initial laser excitation and their positions are recorded.

An alternative approach, providing the Rydberg states have sufficiently long lifetimes, is to allow the Rydberg states to fly unperturbed through the field ionization region (omitting the field ionization pulse) into the flight tube and on to the detector where they are ionized by fields close to the front MCP. The total flight distance from the excitation point to the front MCP is $30.0 \mathrm{~cm}$ and therefore the flight time for an $\mathrm{H}_{2}$ beam moving at $2600 \mathrm{~ms}^{-1}$ would be approximately $115 \mu$ s. Thus only Rydberg states with a lifetime of this order are expected to be observed using such a detection method.

\section{Acceleration/deceleration experiments}

For the acceleration/deceleration experiments (results in Sec. III D) a pair of silver dipole rods (of diameter $2 \mathrm{~mm}$ and center-to-center separation of $5 \mathrm{~mm}$, charged to $\pm 800 \mathrm{~V}$ ) are oriented vertically and perpendicular to the beam direction as shown in Fig. 2(c) and the molecular beam passes centrally between the rods. This produces a field that is horizontal and perpendicular to the beam direction, and the molecules experience an increasing field, as shown in Fig. 4(b), as they move towards the dipole. It is important to apply the dipole field for a fixed time and switch it off well before the Rydberg molecules pass through the dipole, so as to avoid their field ionization, or field-induced predissociation, or passage through level crossings at fields greater than $1000 \mathrm{~V} / \mathrm{cm}$.

As in the deflection experiments, there are two possible modes of field ionization for detection-either a pulsed field is applied across the region between grids 2 and 3, which are mounted immediately behind the dipole and spaced by 1.5 $\mathrm{cm}$, or the molecules are allowed to progress all the way to the detector to be ionized at or near the MCPs.

\section{Classical trajectory simulations}

Classical trajectory simulations were performed by numerical integration of Hamilton's equations of motion using a fourth-order symplectic integrator. ${ }^{25}$ The potential energy gradient at any point in space is determined by combining the calculated spatial field gradient with the Stark map of energy level versus field (see Sec. IIIB) for the populated energy levels in question. In this work the fields were calculated numerically for the dipole rods using an array of discrete charges that were located inside the rods. ${ }^{26}$ The magnitudes of the charges were determined by solving a set of linear equations to produce the correct potentials on the surface of the rods at a series of test points. The fields calculated using this routine are in agreement to better than $1 \%$ with those calculated using commercial software (e.g., SIMION). ${ }^{27}$ Given knowledge of the field as a function of spatial coordinates and the Stark map, the effective potential energy gradient can be determined at any point along the trajectory for input to the integration routine. It is assumed that the motion in the field is adiabatic; i.e., if the jth energy level is initially populated then the system remains in that level, irrespective 


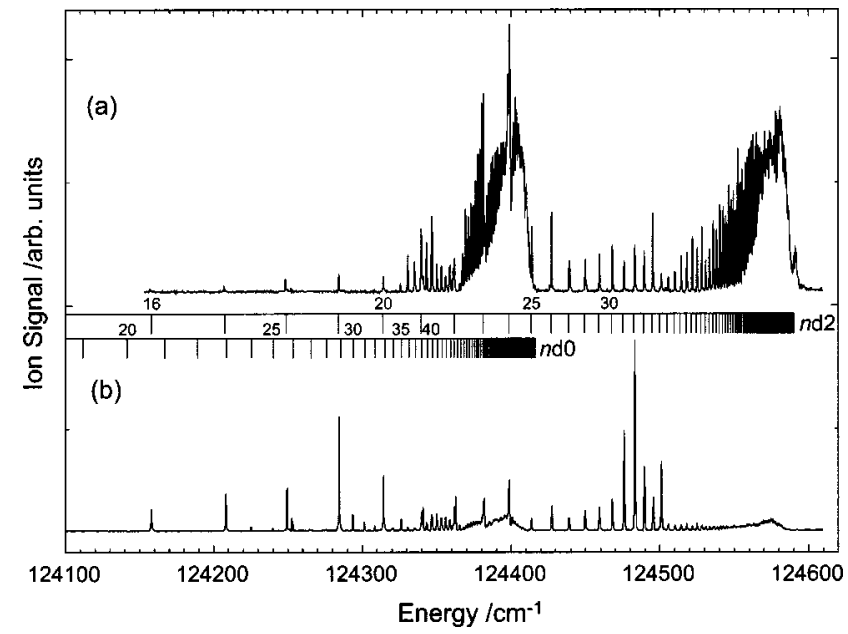

FIG. 5. Zero-field spectrum of $\mathrm{H}_{2}$ obtained by fixing laser 1 to the frequency of the $B-X(0,0) R(0)$ transition populating $J^{\prime}=1$ and scanning the wavelength of laser 2 . Ions are detected by pulsed field ionization after a delay of (a) $3.7 \mu \mathrm{s}$ and (b) $1.2 \mu \mathrm{s}$.

of how the eigenfunction of that level changes with field. If there are avoided crossings between levels then the system is assumed to pass through the crossing adiabatically, as shown in Ref. 20.

In order to simulate the molecular beam conditions, Monte Carlo sampling of the beam-velocity distribution was performed. The velocity distribution emerging from the nozzle orifice was assumed to be described by MaxwellBoltzmann statistics with a temperature of $1 \mathrm{~K}$, superimposed on the molecular beam carrier velocity (assumed 2600 $\mathrm{ms}^{-1}$ for pure $\mathrm{H}_{2}$ ). Only those trajectories passing through a skimmer orifice of $0.9 \mathrm{~mm}$ diameter and through the laser intersection volume are included in the final simulated images. For these purposes the two focused laser beams crossing the molecular beam at right angles are assumed to constitute a square aperture of dimension $0.02 \times 0.02 \mathrm{~mm}$.

\section{RESULTS}

\section{A. Zero-field spectroscopy}

Figure 5(a) shows the Rydberg spectrum of $\mathrm{H}_{2}$ obtained by detecting the total ion signal while tuning the UV laser over the range 291-296 nm with the VUV laser fixed to the $R(0) B\left(\nu^{\prime}=0\right)-X\left(\nu^{\prime \prime}=0\right)$ transition as described above. Two principal series are observed converging to the $N^{+}=0$ and $N^{+}=2$ thresholds. It should be noted that all the observed transitions in Fig. 5(a) are detected through pulsedfield ionization after a delay of $3.7 \mu$ s. A small delayed discrimination field $\left(20 \mathrm{~V} \mathrm{~cm}^{-1}\right)$ is applied to the excitation region so that any ions produced by direct autoionization or fast autoionization will be discriminated against in the detection process. It might therefore seem surprising that at least one of the $(n d 2)_{J}$ series (or both $J=1$ and 2 components if these are unresolved) is observed above the $N^{+}=0$ ionization threshold even though rotational autoionization appears to be energetically possible for these states. However, the $(n d 2)_{1}$ series cannot autoionize because there is no $J=1$ continuum channel of gerade symmetry associated with the

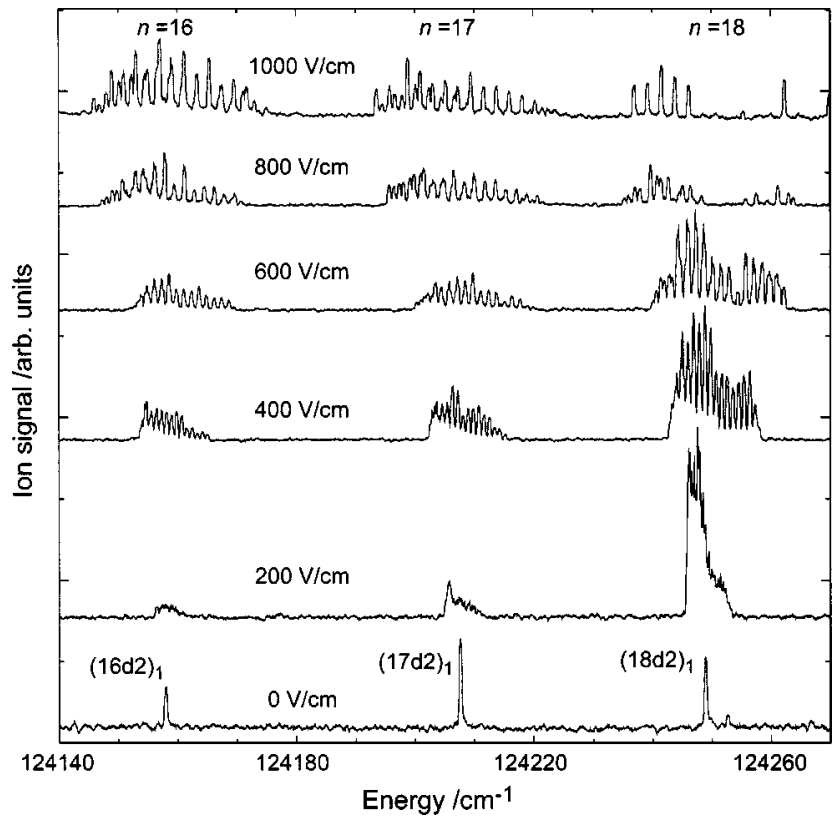

FIG. 6. Pulsed field ionization spectra of $\mathrm{H}_{2}$ obtained in the presence of various homogeneous electric fields in the range $0-1000 \mathrm{~V} / \mathrm{cm}$ shown as a function of the total excitation energy - the sum of the VUV and UV excitation energies.

$N^{+}=0$ threshold (the $(n p 0)_{1}$ channel has ungerade symmetry), and in the absence of a field, the autoionization must obey the $\Delta J=0$ selection rule. It is also interesting to observe that the main series observed in the region $\approx 200 \mathrm{~cm}^{-1}$ below the $N^{+}=0$ threshold (i.e., near $124200 \mathrm{~cm}^{-1}$ ) is not the $(n d 0)_{2}$ series converging to that threshold but is a series converging to the $N^{+}=2$ threshold. The $N^{+}=0$ Rydberg states below $n=35$ apparently do not survive the $3.7 \mu \mathrm{s}$ delay time before field ionization. Previous spectroscopic studies ${ }^{28-30}$ have shown that there is a fast predissociation of the $(n d 0)_{2},(n d 2)_{2}$, and $(n s 2)_{2}$ series in this region presumably due to the presence of a broad strongly predissociated $J=2$ interloper state of high $\nu^{+}$and low $n$ (see for example Ref. 30, Fig. 5), and it is only the ( $n d 2)_{1}$ series that survives. As shown in Fig. 5(b), if the delay time before field ionization is shortened to $1.2 \mu \mathrm{s}$, the $(n d 0)_{2}$ series extends further down to around $n=30$, and the $(n d 2)_{1}$ series also gains intensity in the same spectral region. To summarize, the main series observed at zero field are the $(n d 0)_{2}$ and the $(n d 2)_{1}$ series with the latter dominating.

\section{B. Stark spectroscopy and simulation}

Figure 6 shows the region of the spectrum recorded in the range of laser $2, \lambda=295.0-293.9 \mathrm{~nm}$ corresponding to a total excitation energy $124140-124270 \mathrm{~cm}^{-1}$, with the excitation carried out in the presence of a series of different homogeneous electric fields. As above, the ions are detected by field ionization after a delay of $3.7 \mu \mathrm{s}$. The $(16-18 d 2)_{1}$ lines at zero field are observed to split out into hydrogenic Stark manifolds, resulting from the admixture of the $n d$ states into the $N^{+}=2$ high- $\ell$ zero-quantum-defect states of the same $n$. Figure 7(a) shows a simulated Stark map, illustrating how the energy levels for $n=17, N^{+}=2, M_{J}=0$ vary 

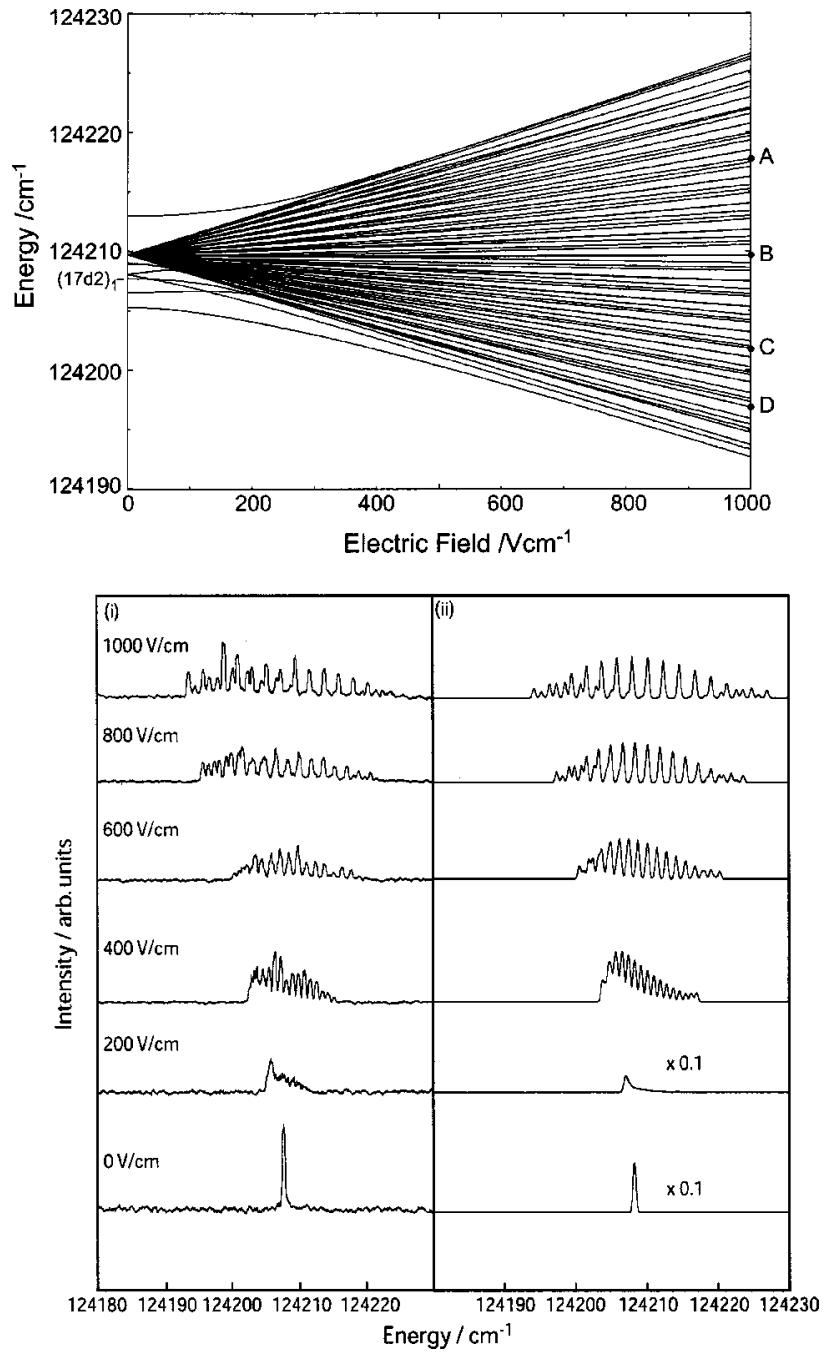

FIG. 7. (a) Stark map for $n=17, N^{+}=2$, and $M_{J}=0$ calculated as described in the text. The closed circles labeled $A-D$ indicate the levels selected for the deflection experiments. (b) Comparison between (i) experimental and (ii) calculated Stark spectrum of the $N^{+}=2, n=17$. Stark manifold. The simulation has been convoluted with a Gaussian laser bandwidth full width half maximum of $0.5 \mathrm{~cm}^{-1}$.

with electric field, while Fig. 7(b) compares the simulated and experimental spectra for fields of $0-1000 \mathrm{~V} / \mathrm{cm}$. The majority of the levels, for which the eigenfunctions are linear combinations of high- $\ell$ states $(\ell>2)$, show the linear Stark effect, which is characteristic of the $\mathrm{H}$ atom and is also typically shown by high- $\ell$ Rydberg states in all atoms and molecules studied to date (see for example Refs. 31-36). Quadratic Stark shifts are characteristic of the lower- $\ell$ states at low fields, although these states merge ultimately into the high- $\ell$ manifolds and the Stark shifts for these levels become linear also.

The calculated Stark map was obtained by diagonalization of the effective Hamiltonian with a basis set of the form $\left\{\nu^{+} N^{+} n \ell J M_{J}\right\} \cdot{ }^{31,36,37}$ As stated above the $J, M_{J}$ quantum numbers are equivalent to $N, M_{N}$ if it is assumed that only singlet states are involved. Only $\nu^{+}=0, N^{+}=0$ and 2 states are included (hence we drop the $\nu^{+}$subscript hereafter), and the calculations for the $n=17, N^{+}=2$ Stark manifold included the basis functions with $n=16$ to $18,\left(N^{+}=2\right)$ and
$n=21-27,\left(N^{+}=0\right)$. The Hamiltonian is of the form

$$
H=H_{0}+H_{\text {stark }},
$$

where the matrix elements of $H_{\text {stark }}$, the Stark Hamiltonian, (diagonal in $N^{+}$, off-diagonal in $\ell$ ) are given in the Hund's case (d) basis by Eq. (1) of Ref. 37 with the radial integral evaluated using the Coulomb approximation as given in Refs. 31, 32, and 36.

In the experimental setup the laser polarizations are perpendicular to each other and to the Stark field. Defining the space quantization axis as the Stark field direction leads to the selection rule $\Delta M_{J}=0, \pm 2$, for the overall two-step excitation and therefore it is the states with $M_{J}=0, \pm 2$ that are populated (in this quantization frame) starting from $J^{\prime \prime}=0$ in the ground state. An important difference for the $M_{J}=0$ states compared to all other $M_{J}$ states is that the selection rule for the Stark matrix elements is $\Delta J= \pm 1$ for $M_{J}=0$, whereas it is $\Delta J=0, \pm 1$ for all other $M_{J}$ values.

The diagonal matrix elements of the zero-field Hamiltonian are given by

$$
\begin{aligned}
& \left\langle N^{+} n \ell J M_{J}\left|H_{0}\right| N^{+} n \ell J M_{J}\right\rangle \\
& \quad=I\left(N^{+}\right)+\Sigma_{\Lambda}\left\langle N^{+} \ell \mid \Lambda\right\rangle^{J} E_{n \ell \Lambda}\left\langle\Lambda \mid N^{+} \ell\right\rangle^{J},
\end{aligned}
$$

where $I\left(N^{+}\right)$is the ionization threshold for a given $N^{+}$, and $E_{n \ell \Lambda}$ represents the electronic energy of a state $|n \ell \Lambda\rangle$ in the pure Hund's case (b) representation; $E_{n \ell \Lambda}=-\mathcal{R} /(n$ $\left.-\mu_{\ell \Lambda}\right)^{2}$ where $\mu_{\ell \Lambda}$ is the eigenchannel quantum defect and $\mathcal{R}$ is the Rydberg constant. $\left\langle N^{+} \ell \mid \Lambda\right\rangle^{J}$ is the transformation coefficient from Hund's case (d) to Hund's case (b) as given in Ref. 36. Explicit expressions for Eq. (2) are given in Table II of Ref. 29.

The only off-diagonal matrix elements of $H_{0}$ included are those involving $\Delta N^{+}=2, \Delta \ell=0$, and $\ell<3$; i.e., the coupling between the $(n p 0)_{1}$ and $(n p 2)_{1}$ channels and between the $(n d 0)_{2}$ and $(n d 2)_{2}$ channels are considered, these elements being given as Ref. 36:

$$
\begin{aligned}
\left\langle N^{+}\right. & \left.n \ell J M_{J}\left|H_{0}\right| N^{+\prime} n^{\prime} \ell J M_{J}\right\rangle \\
& =\sum_{\Lambda}\left\langle N^{+} \ell \mid \Lambda\right\rangle^{J} H_{\ell \Lambda}\left\langle\Lambda \mid N^{+\prime} \ell\right\rangle^{J},
\end{aligned}
$$

where $H_{\ell \Lambda}=-\pi \mu_{\ell \Lambda} /\left(\nu^{3 / 2} \nu^{\prime 3 / 2}\right)$.

The matrix elements of $H_{0}$ off-diagonal in $\ell$, notably those between the $s$ and the $d$ series, are ignored because, as shown by Jungen and Ross, ${ }^{38}$ the $s-d$ electronic coupling is very small, and maximized in the region of internuclear distance around 4 a.u. This region is essentially outside the range of the core vibrational wave function in $\nu^{+}=0$. However, as pointed out by Rottke and Welge, ${ }^{30}$ the $s$ and $d$ eigenchannel quantum defects effectively contain a small adjustment as a result of their adiabatic treatment of the $s-d$ interaction. The $p-f$ interaction is expected to be even weaker and is also ignored. The values for the quantum defects used in the calculation were $\mu(s \sigma)=0.884,{ }^{30} \mu(p \sigma)$ $=0.182,{ }^{31} \quad \mu(p \pi)=-0.073,{ }^{31} \quad \mu(d \sigma)=0.071, \quad \mu(d \pi)$ $=0.045$ and $\mu(d \delta)=-0.017,{ }^{30}$ and the ionization thresh- 


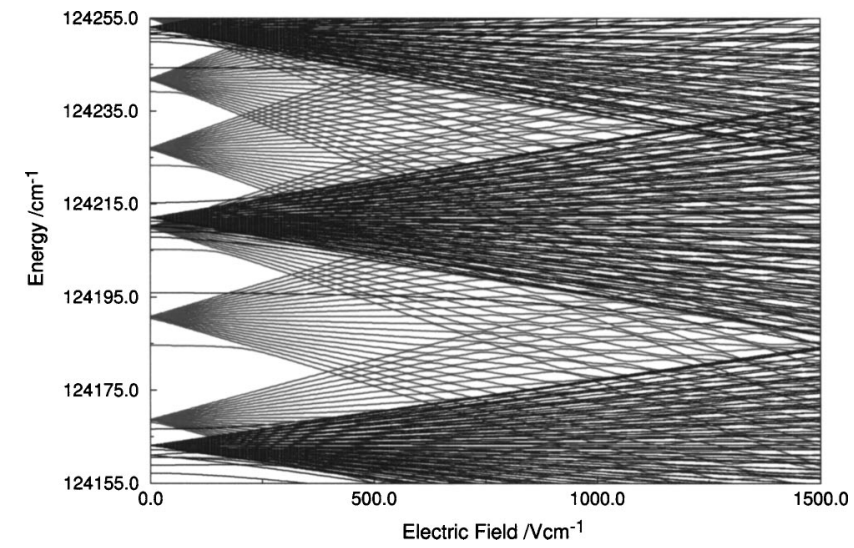

FIG. 8. Stark map including all $N^{+}=0(n=21-26)$ and $N^{+}=2(n$ =16-18) $M_{J}=0$ states (the $N^{+}=2$ levels are shown with bolder lines).

olds are equal to $I(0)=124417.507 \mathrm{~cm}^{-1},{ }^{24}$ and $I(2)$ $=124591.743 \mathrm{~cm}^{-1}$ (using the rotational energy of $\mathrm{H}_{2}^{+}$from Ref. 39).

The intensity of a transition to the final state $i$, one of the eigenstates of the Hamiltonian, is calculated using the coefficients $c_{i j}$ in the expansion of state $i$ in terms of the basis functions $j$ :

$$
\begin{aligned}
& \Psi_{i}=\sum_{j} c_{i j} \phi_{j}^{0}, \\
& I_{i} \propto\left|\sum_{j} c_{i j}\langle j|\mu| 0\rangle\right|^{2},
\end{aligned}
$$

where $j$ represents $\left\{N^{+}, n, l, J, M_{J}\right\}$ and 0 is the intermediate state from which the transition originates. A good representation of the spectrum was only obtained by setting all transition moments $\langle j|\mu| 0\rangle$ to zero except for that in which $j$ corresponds to the $(n d 2)_{1}$ basis function. As this state does not have an $M_{J}=2$ component, it is only the $M_{J}=0$ states that are populated through intensity borrowing with this state. It should be noted that the experimental spectra shown in Figs. 5-7 are not only determined by the transition intensities but also by the decay of certain states, given the $3.7 \mu \mathrm{s}$ delay before detection by field ionization. Those states with short lifetimes may not appear in the experimental spectra. The apparent unimportance of the $(n d 2)_{2}, M_{J}=0$ or 2 transition moments, or those involving the $n s$ functions may partly (or totally) reflect the shorter lifetimes of states with a significant admixture of these basis functions.

It might be expected however that the application of a field could cause sufficient mixing of states to induce the decay of the states that are longest lived at zero field. For example if the long lived $(n d 2)_{1} M_{J}=0$ states were mixed with the $(n d 2)_{2}$ or $(n s 2)_{2}$ states then fast decay of the former could ensue. It might also be expected that there would be complications in this spectroscopic region arising from the crossings of $N^{+}=0$ Stark manifolds with the $N^{+}$ $=2$ manifolds. Figure 8 shows a full Stark map in which the $N^{+}=0$ manifolds for $n=21-26$ are included, and the $N^{+}$ $=2$ manifolds from $n=16$ to 18 . In the context of a deflection or deceleration experiment it would need to be understood whether the system passed through the multiple crossings of these Stark manifolds in a diabatic or adiabatic manner, as discussed in detail in Ref. 20.

In fact it turns out that the $M_{J}=0$ states populated in the experiments carried out here are a special case (compared to other $M_{J}$ values), in that the Stark Hamiltonian is block diagonalized into two sets of noninteracting states, as shown in Table I. The consequence is that the potentially undesirable $N^{+}$-mixing and $J$-mixing effects do not actually occur for the states in the upper block. Not only is there no zero-field coupling of the $(n d 2)_{1}$ state (and other $N^{+}=2$ states in the upper block) to the $N^{+}=0$ channels, but also the $\Delta J= \pm 1$, $\Delta N^{+}=0$ selection rule for field-induced mixing of $M_{J}=0$ states means that there is no mechanism for field-induced

TABLE I. Hamiltonian matrix for the $M_{J}=0, \ell=0-3, N^{+}=0-2$ Rydberg states in the presence of an electric field, showing which elements are nonzero and hence the block-diagonal structure of the matrix. $H_{0}$ indicates a nonzero matrix element of the zero-field Hamiltonian while $H_{\text {stark }}$ indicates a nonzero element

\begin{tabular}{|c|c|c|c|c|c|c|c|c|c|c|c|c|c|c|c|c|c|c|}
\hline & $(p 2)_{2}$ & $(d 2)_{1}$ & $(d 2)_{3}$ & $(f 2)_{2}$ & $(f 2)_{4}$ & $(s 0)_{0}$ & $(s 2)_{2}$ & $(p 0)_{1}$ & $(p 2)_{1}$ & $(p 2)_{3}$ & $(d 0)_{2}$ & $(d 2)_{0}$ & $(d 2)_{2}$ & $(d 2)_{4}$ & $(f 0)_{3}$ & $(f 2)_{1}$ & $(f 2)_{3}$ & $(f 2)_{5}$ \\
\hline p2) & $H_{0}$ & $H_{\text {stark }}$ & $H_{\text {stark }}$ & $\left(H_{0}\right)$ & $\ldots$ & $\ldots$ & $\ldots$ & $\ldots$ & $\ldots$ & $\ldots$ & $\ldots$ & $\ldots$ & $\ldots$ & $\ldots$ & $\ldots$ & $\ldots$ & $\ldots$ & $\ldots$ \\
\hline 12) & $H_{\text {stark }}$ & $H_{0}$ & . & $H_{\text {stark }}$ & $\ldots$ & $\ldots$ & $\ldots$ & $\ldots$ & $\cdots$ & $\ldots$ & $\ldots$ & $\ldots$ & $\ldots$ & $\ldots$ & $\ldots$ & $\ldots$ & $\ldots$ & $\ldots$ \\
\hline$d 2)_{3}$ & $H_{\text {stark }}$ & $\ldots$ & $H_{0}$ & $H_{\text {stark }}$ & $H_{\text {stark }}$ & $\cdots$ & $\ldots$ & $\cdots$ & $\cdots$ & $\cdots$ & $\cdots$ & $\cdots$ & $\cdots$ & $\cdots$ & $\cdots$ & $\cdots$ & $\ldots$ & $\cdots$ \\
\hline 2$)_{2}$ & $\left(H_{0}\right)$ & $H_{\text {stark }}$ & $H_{\text {stark }}$ & $H_{0}$ & $\cdots$ & $\cdots$ & $\ldots$ & $\cdots$ & $\ldots$ & $\cdots$ & $\cdots$ & $\cdots$ & $\cdots$ & $\cdots$ & $\cdots$ & $\cdots$ & $\ldots$ & $\cdots$ \\
\hline$f 2)_{4}$ & $\ldots$ & .. & $H_{\text {stark }}$ & $\cdots$ & $H_{0}$ & $\ldots$ & $\ldots$ & $\ldots$ & $\ldots$ & $\ldots$ & $\ldots$ & $\ldots$ & $\ldots$ & $\ldots$ & $\ldots$ & $\ldots$ & $\ldots$ & $\ldots$ \\
\hline$s 0)_{0}$ & $\ldots$ & $\ldots$ & $\cdots$ & $\ldots$ & $\cdots$ & $H_{0}$ & $\ldots$ & $H_{\text {stark }}$ & $\ldots$ & $\ldots$ & $\ldots$ & $\left(H_{0}\right)$ & $\ldots$ & $\ldots$ & $\ldots$ & $\ldots$ & $\ldots$ & $\ldots$ \\
\hline$(s 2)_{2}$ & $\cdots$ & $\cdots$ & $\cdots$ & $\cdots$ & $\cdots$ & $\cdots$ & $H_{0}$ & $\cdots$ & $H_{\text {stark }}$ & $H_{\text {stark }}$ & $\left(H_{0}\right)$ & $\ldots$ & $\left(H_{0}\right)$ & $\cdots$ & $\cdots$ & $\cdots$ & $\cdots$ & $\cdots$ \\
\hline$(p 0)_{1}$ & $\ldots$ & $\ldots$ & $\ldots$ & $\ldots$ & $\ldots$ & $H_{\text {stark }}$ & $\ldots$ & $H_{0}$ & $H_{0}$ & $\ldots$ & $H_{\text {stark }}$ & $\ldots$ & $\ldots$ & $\ldots$ & $\ldots$ & $\left(H_{0}\right)$ & $\ldots$ & $\ldots$ \\
\hline$(p 2)_{1}$ & $\ldots$ & $\ldots$ & $\ldots$ & $\ldots$ & $\cdots$ & ... & $H_{\text {stark }}$ & $H_{0}$ & $H_{0}$ & $\ldots$ & . & $H_{\text {stark }}$ & $H_{\text {stark }}$ & $\ldots$ & $\ldots$ & $\left(H_{0}\right)$ & $\ldots$ & $\ldots$ \\
\hline$p 2)_{3}$ & $\cdots$ & $\cdots$ & $\cdots$ & $\cdots$ & $\cdots$ & $\cdots$ & $H_{\text {stark }}$ & $\cdots$ & $\cdots$ & $H_{0}$ & $\ldots$ & $\cdots$ & $H_{\text {stark }}$ & $H_{\text {stark }}$ & $\left(H_{0}\right)$ & $\cdots$ & $\left(H_{0}\right)$ & $\cdots$ \\
\hline$(d 0)_{2}$ & $\cdots$ & $\ldots$ & $\cdots$ & $\ldots$ & $\cdots$ & $\ldots$ & $\left(H_{0}\right)$ & $H_{\text {stark }}$ & $\ldots$ & $\cdots$ & $H_{0}$ & $\ldots$ & $H_{0}$ & .. & $H_{\text {stark }}$ & $\ldots$ & $\ldots$ & . \\
\hline$(d 2)_{0}$ & $\ldots$ & $\ldots$ & $\ldots$ & $\ldots$ & $\ldots$ & $\left(H_{0}\right)$ & $\ldots$ & . & $H_{\text {stark }}$ & $\ldots$ & $\ldots$ & $H_{0}$ & $\ldots$ & $\ldots$ & ... & $H_{\text {stark }}$ & $\ldots$ & $\ldots$ \\
\hline$(d 2)_{2}$ & $\cdots$ & $\cdots$ & $\cdots$ & $\cdots$ & $\cdots$ & $\cdots$ & $\left(H_{0}\right)$ & $\cdots$ & $H_{\text {stark }}$ & $H_{\text {stark }}$ & $H_{0}$ & $\cdots$ & $H_{0}$ & $\cdots$ & $\cdots$ & $H_{\text {stark }}$ & $H_{\text {stark }}$ & $\cdots$ \\
\hline$(d 2)_{4}$ & $\cdots$ & $\cdots$ & $\cdots$ & $\ldots$ & $\cdots$ & $\cdots$ & $\cdots$ & $\cdots$ & .. & $H_{\text {stark }}$ & $\cdots$ & $\cdots$ & $\cdots$ & $H_{0}$ & $\cdots$ & ‥ & $H_{\text {stark }}$ & $H_{\text {stark }}$ \\
\hline$f 0$ & $\ldots$ & $\ldots$ & $\ldots$ & $\ldots$ & $\ldots$ & $\ldots$ & $\ldots$ & $\ldots$ & $\ldots$ & $\left(H_{0}\right)$ & $H_{\text {stark }}$ & $\ldots$ & $\ldots$ & $\ldots$ & $H_{0}$ & $\ldots$ & $H_{0}$ & \\
\hline$(f 2)_{1}$ & $\cdots$ & $\cdots$ & $\cdots$ & $\cdots$ & $\cdots$ & $\cdots$ & $\cdots$ & $\left(H_{0}\right)$ & $\left(H_{0}\right)$ & $\ldots$ & $\cdots$ & $H_{\text {stark }}$ & $H_{\text {stark }}$ & $\cdots$ & $\cdots$ & $H_{0}$ & $\cdots$ & $\ldots$ \\
\hline$(f 2)_{3}$ & $\ldots$ & $\ldots$ & $\ldots$ & $\ldots$ & $\cdots$ & $\ldots$ & $\ldots$ & $\cdots$ & $\cdots$ & $\left(H_{0}\right)$ & $\ldots$ & ... & $H_{\text {stark }}$ & $H_{\text {stark }}$ & $H_{0}$ & $\cdots$ & $H_{0}$ & $\cdots$ \\
\hline$(f 2)_{5}$ & $\cdots$ & $\cdots$ & $\cdots$ & $\cdots$ & $\cdots$ & $\cdots$ & $\cdots$ & $\cdots$ & $\cdots$ & $\cdots$ & $\cdots$ & $\cdots$ & $\cdots$ & $H_{\text {stark }}$ & $\cdots$ & $\cdots$ & $\cdots$ & $H_{0}$ \\
\hline
\end{tabular}
of the electric field perturbation. 
coupling to the $N^{+}=0$ states. This behavior is in contrast to the $M_{J}=1$ states; for example, the added possibility of $\Delta J$ $=0$ coupling by the field means that the $(n d 2)_{1}$ state can couple in the field to the $(n p 2)_{1}$ state, which is strongly mixed by the zero-field Hamiltonian with $(n p 0)_{1}$. Thus the same block diagonal structure does not occur for the $M_{J}$ $=1$ states. In a similar vein, the $M_{J}=1$ states can undergo a second-order field-induced mixing of the type $(n d 2)_{1}-(n p 2)_{1}-(n d 2)_{2}$ which leads to facile $J$ mixing of the $(n d 2)_{J}$ states. Such $J$ mixing is not possible for $M_{J}=0$ states for which the $(n d 2)_{1}-(n p 2)_{1}$ coupling is forbidden, and thus the long-lived $J=1$ states are protected from the field-induced predissociation that would arise if they were coupled to the short-lived $(n d 2)_{2}$ states.

The important conclusion is that the $M_{J}=0$ states we observe in the Stark spectrum after a time delay of several microseconds are those which arise from diagonalization of the upper block of Table I. These states have metastability because of the restricted couplings, and this feature is of importance in the success of the present experiments.

\section{Deflection experiments}

In the first experiments using the horizontal dipole setup, described in Sec. II B 2 [Fig. 2(b)], the deflecting inhomogeneous field was applied for $7 \mu$ s after Rydberg excitation and then switched off. After approximately $12 \mu$ s the Rydberg molecules entered the field ionization region between grids 2 and 3 (Fig. 2) and were field ionized and accelerated to the detector. Two-dimensional images showing the deflected positions of the molecules were recorded using the CCD camera.

Figure 9(b) shows a series of images obtained in which different Stark states of the $n=16$ and 17 manifolds were populated; these states are labeled $A-M$ in the Stark spectrum shown in Fig. 9(a). The spectrum is broader nearer the outside of the Stark manifolds because the distribution of fields experienced by the molecules in the finite excitation volume correlates with the greatest spread of energy for the levels with the largest Stark shift. Each of the 13 strips shown in Fig. 9 corresponds to a separate image recorded by collecting the signal over approximately 512 laser pulses. The square shape of each image results from the square shape of the laser interaction cross section under the focusing conditions of the experiment. However, there is apparently some magnification of the size of these images as the ions pass through the second extraction grid arising from the significant field gradient across the apertures of the grid leading to a microscopic lensing effect. It can be seen that there is, as expected, an upward deflection on average if one of the blueshifted low-field seeking states is populated (e.g., states $F$, $M)$ and a downward deflection if a redshifted high-field seeking state is populated (e.g., $A, G$ ). The change between states $F$ and $G$ (from the $n=16$ blueshifted state to the adjacent $n=17$ redshifted state) is especially noticeable in the figure.

In these images, the range of mean deflections observed covers only about $2 \mathrm{~mm}$, and is less than the overall spread of positions in a single image, although this spread is in part due to lensing at the second extraction grid. In order to observe larger deflections we allowed the Rydberg states to fly
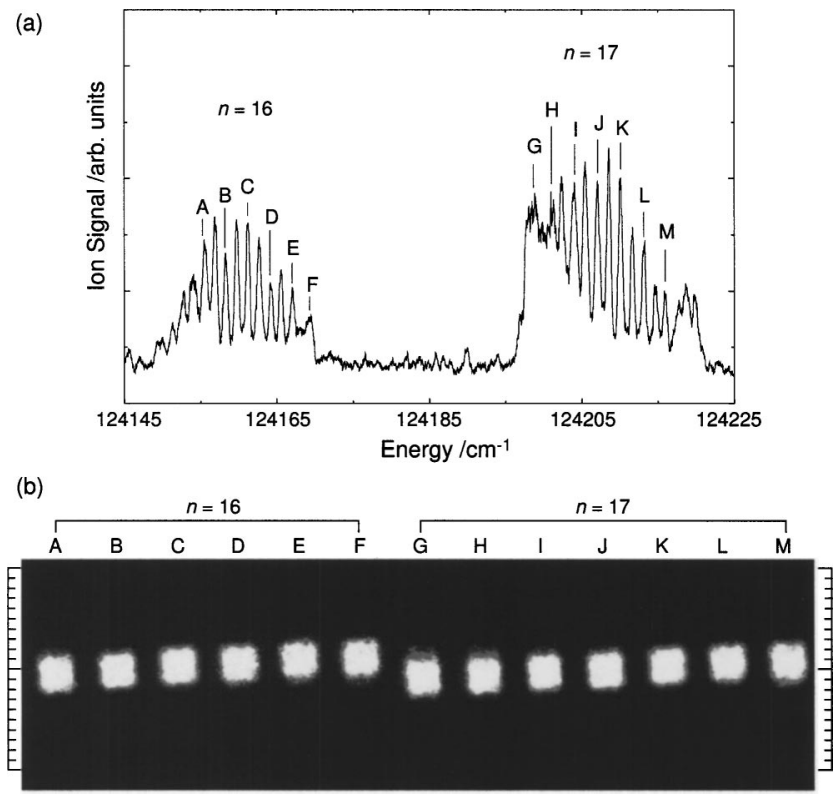

FIG. 9. (a) Pulsed field ionization spectrum of $\mathrm{H}_{2}$ recorded in the presence of the inhomogeneous field produced by the dipole in the horizontal configuration. The field at the point of excitation is approximately $600 \mathrm{~V} \mathrm{~cm}^{-1}$ and the observed $n=16,17$ high $\ell$ Stark manifolds correspond to the $\nu^{+}$ $=0, N^{+}=2$ core state. (b) Images showing the distribution of arrival positions of the $\mathrm{H}_{2}$ Rydberg molecules at the detector after deflection in the dipole field for $7 \mu \mathrm{s}$, for population of the Rydberg levels labeled $A-M$ in (a). The molecules are field ionized between grids 2 and 3 and detected after 10-11 $\mu$ s flight time. The vertical scales show $1 \mathrm{~mm}$ intervals.

all the way to the detector $(\approx 30 \mathrm{~cm})$ before field ionization, as described in Sec. II B 2, so that the time of flight would be greater. This approach also eliminates the lensing effect, as the ions do not pass through a field gradient on traversing grid 3. Some of the images obtained were first reported in Ref. 19; those recorded for four different Stark states denoted $A-D$ after applying the dipole field for $1 \mu \mathrm{s}$ are shown in Fig. 10(a), with the states being as labeled in the Stark map shown in Fig. 7(a). The field at the point of excitation is 684 $\mathrm{V} / \mathrm{cm}$. These and more data are displayed in a more quantitative form in Fig. 11, in which the positions of the centroid averages of the images are plotted (open circles, triangles

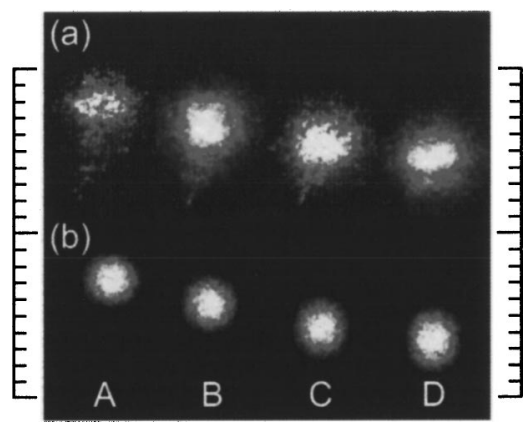

FIG. 10. (a) Images showing the distribution of arrival positions of the $\mathrm{H}_{2}$ Rydberg molecules at the detector after deflection in the dipole field for 1 $\mu$ s, for population of the Rydberg levels labeled $A-D$ in Fig. 7(a). The vertical scale shows $1 \mathrm{~mm}$ intervals. The molecules are field ionized at the detector after a flight time of $110 \mu \mathrm{s}$. (b) 10000 point Monte Carlo classical trajectory simulations of deflection corresponding to the experimental conditions in (a) with a beam temperature of $1 \mathrm{~K}$. 


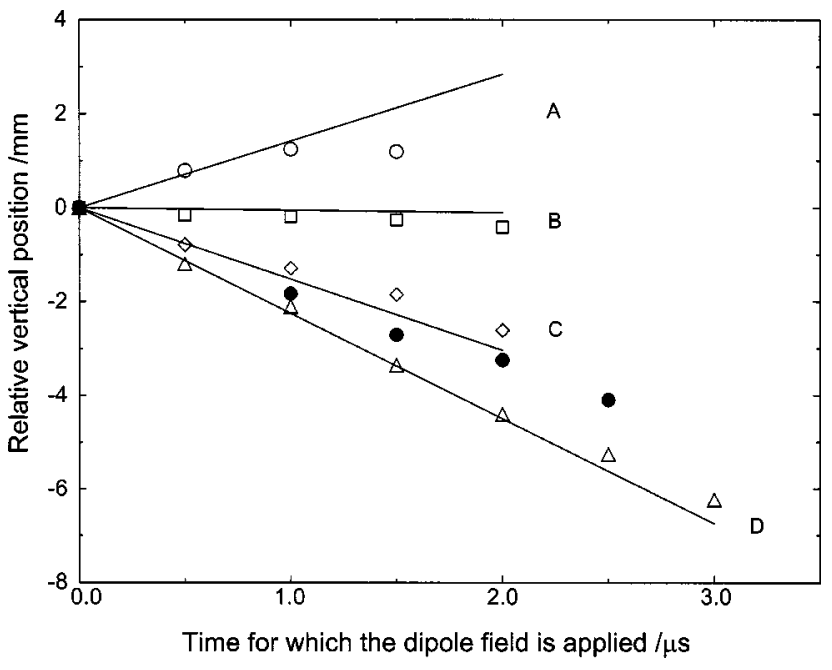

FIG. 11. Mean deflection (position of the image centroid average) as a function of duration of inhomogeneous field application for various levels. The experimental points for population of levels $A-D$ are shown using open triangles, circles, etc. The bold lines show the calculated deflection of an ideal trajectory traveling along the central beam axis with a velocity of 2600 $\mathrm{ms}^{-1}$. The points shown as closed circles correspond to the experimentally measured mean deflection when populating the $(17 d 2)_{1}$ level in the absence of a field and then switching on the deflecting field $0.5 \mu$ s later.

etc.) as a function of the length of time over which the deflection field is applied for the four Stark states $A-D$. The maximum deflection observed is about $6 \mathrm{~mm}$ for a field application of $3.0 \mu \mathrm{s}$ and flight time of $110 \mu \mathrm{s}$, implying a perpendicular velocity component in the field-free flight region of $\approx 60 \mathrm{~ms}^{-1}$.

We have carried out trajectory simulations to model the observed behavior. In Fig. 10(b), simulated images obtained from Monte Carlo trajectory calculations (10000 trajectories) are shown for the single Stark levels labeled $A-D$ in Fig. 7(a) and for the same magnitude and duration of the applied field as in the experimental results. The resolution of the experimental spectra is such that more than one closely spaced level could be populated in the excitation processes corresponding to images labeled $A-D$. However, the simulations of the Stark spectra indicate that a transition to one level dominates the observed spectral line in each case, and therefore population of a single level is assumed for the trajectory calculations. The Monte Carlo sampling of the initial velocities is discussed in Sec. II C. A good reproduction of the experimental results is achieved in these simulations as shown in the figure.

In Fig. 11 the calculated deflections of "model" trajectories are shown for the four different Stark levels. For these ideal trajectories, the molecule is set off traveling along the axis of the molecular beam at the mean molecular beam velocity $\left(2600 \mathrm{~ms}^{-1}\right)$ and the pulsed dipole field is applied for variable durations. Figure 11 shows that the data for level $D$ is well reproduced by the simulations, whereas differences are apparent for the low-field seeking state $A$. Experimentally the centroid-averaged image position for Stark state $A$ does not change linearly with the time of field application, but levels off, a feature which is not reproduced in the simulations. Because the change in vertical position of the mol- ecules during the 0-2 $\mu$ s application of the dipole field is very small, the field gradient should not change markedly during the period of acceleration, and a linear trend is predicted by the calculations if the field gradient is constant. However, it should also be noted that the signal levels are significantly lower for the low-field seeking states such as $A$, and a weak underlying undeflected component, which appears to be present in all images, seems to become more prominent in the observed images for level $A$, shifting the mean position. The reason for the existence of this undeflected component remains a matter for speculation. It is probable that blackbody radiation stimulated transitions occur between the Rydberg energy levels during the $\mu$ s duration field application and it is possible that this leads to conversion between different components of the Stark manifold or states of different $n$ in such a way that the low-field seeking character is lost or diminished. However, the strongest blackbody-induced transitions tend to conserve the $k$ quantum number, and hence also conserve the deflection behavior, and therefore the origin of this undeflected signal needs further investigation.

The relative weakness of the deflected signal due to the blueshifted low-field seeking Stark states suggests a shorter lifetime for these states. As stated in our preliminary report ${ }^{19}$ a surprising feature of this experiment is that any of the Stark components actually survive the $110 \mu$ s trajectories to the detector. The observed time of flight is only $5 \%$ less than that predicted for the molecules traveling at the expected beam velocity of $2600 \mathrm{~ms}^{-1}$, so we can be sure that these molecules are not ionized until they are less than $2 \mathrm{~cm}$ from the detector. It should be noted that autoionization does not occur for these levels, which all lie in energy below the adiabatic ionization threshold. Previous work has suggested that the lifetimes of some of the $\ell<3$ Rydberg series with respect to predissociation are on the order of $\mu$ s or less. ${ }^{28}$ The strong mixing of $\ell$ character of the Rydberg states in the presence of the dipole field would be expected to lead to longer lifetimes for all the Stark states relative to the zero-field low- $\ell$ states. Nevertheless, the typical lifetime for all Stark states would not be expected to be much longer than $10 \mu \mathrm{s}$. A key point in the present experiments is that the field is switched off after, at most, $7 \mu$ s and the majority of the flight is field free. As the field is switched off, the molecules will follow the Stark map adiabatically. Figure 7(a) shows that the populated states $A-D$ correlate adiabatically with the long-lived, zeroquantum-defect, high- $\ell$ states at low field. As shown previously by Schlag and co-workers for $\mathrm{NO},{ }^{40}$ and in our own calculations, ${ }^{41}$ the "locking" of a proportion of the population into high- $\ell$ states means that the excited molecules become metastable with respect to predissociation and other decay processes. We cannot rule out the possibility that the Rydberg states undergo a change of principal quantum number (or other quantum numbers) during the $110 \mu$ s flight time, possibly due to microwave or far-infrared transitions induced by the blackbody radiation. Our detection method will not be sensitive to the occurrence of such transitions provided that the final states of these transitions have similar properties to those of the initial states. Further discussion and experimental results relating to the decay dynamics of these 


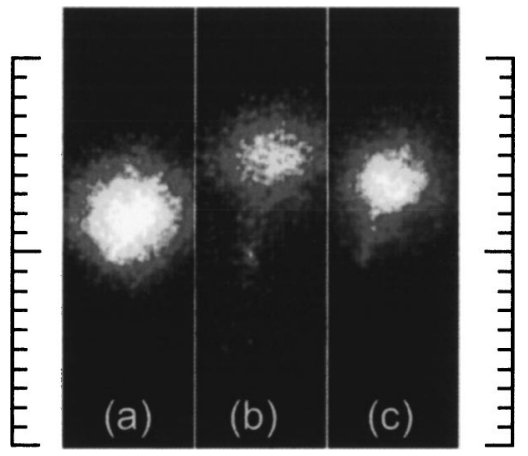

FIG. 12. Images showing deflection of Rydberg molecules when populating the $(17 d 2)_{1}$ level in the absence of a field and then switching on the deflecting field $0.5 \mu \mathrm{s}$ later for a duration of (a) $2.0 \mu \mathrm{s}$, (b) $0 \mu \mathrm{s}$, (c) $1.0 \mu \mathrm{s}$. The vertical scale shows $1 \mathrm{~mm}$ intervals.

states will be presented in a future paper, ${ }^{42}$ while related studies on argon Rydberg states are described in Ref. 20.

In all of the deflection experiments described above, the Rydberg excitation took place in the presence of an electric field and therefore the initial state populated was a Stark eigenstate. Figure 12 shows the deflected images obtained in an experiment in which the excitation takes place in zero field and then the inhomogeneous field due to the dipole is rapidly switched on after a delay of $500 \mathrm{~ns}$. The centroid average positions of the images are shown in Fig. 11 (filled-in circles) as a function of applied field duration. A sizeable deflection, comparable with that observed when populating an extreme high-field seeking state of the nearby manifold, is still clearly observed in this situation. The state populated in zero field is the $(17 d 2)_{1}$ state and as shown in Fig. 7(a), this is located slightly on the low-energy side of the manifold. As the field rises it eventually evolves into a high-field seeking level undergoing the linear Stark effect and thus a downward deflection towards the dipole would be predicted and is indeed observed. At high field, the Starkmap gradient of the level to which $(17 d 2)_{1}$ state correlates adiabatically is greater than that for level $D$ and therefore a greater deflection might be predicted. In fact the magnitude of the deflection observed is actually between that for levels $C$ and $D$. A possible explanation would be that the system does not pass through every avoided crossing adiabatically when the field is rapidly switched on $\left(0-\approx 600 \mathrm{~V} \mathrm{~cm}^{-1}\right.$ with a $10 \mathrm{~ns}$ rise time). This would be in contrast to the completely adiabatic behavior observed when the field changes relatively slowly as the molecules move through an inhomogeneous field. ${ }^{20}$

\section{Deceleration/acceleration experiments}

Deceleration/acceleration experiments were carried out using the dipole mounted vertically as described in Sec. II B 3 [Fig. 2(c)]. In this case the field inhomogeneity is predominantly along the molecular beam axis rather than perpendicular to it. Thus, there is no off-axis deflection and the 2D images recorded at the position-sensitive detector do not change significantly in size or position with the applied field or with Stark state even when the flight time is $>100 \mu \mathrm{s}$. Significant changes are observed, however, in the time-of-

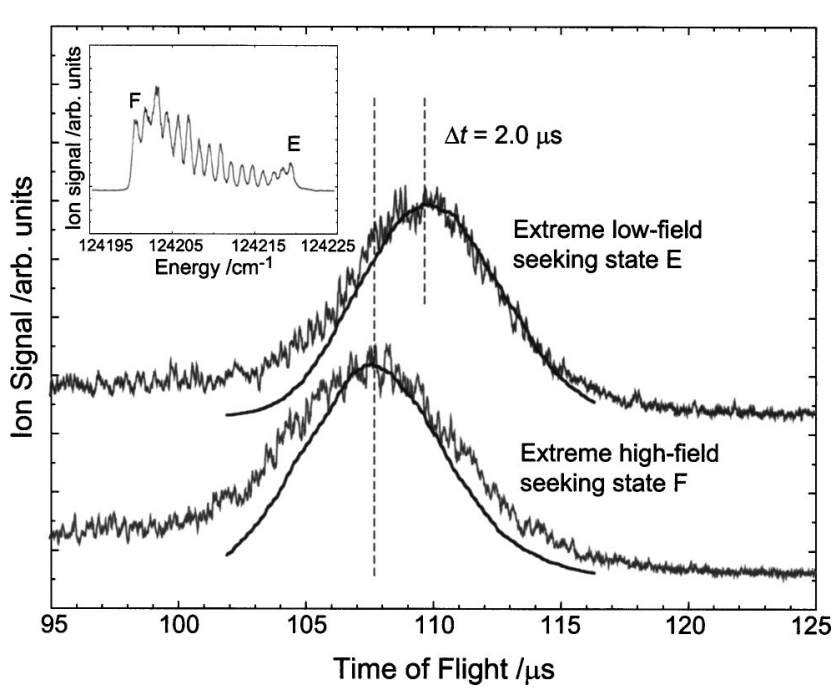

FIG. 13. Time-of-flight distribution of the Rydberg states at the detector for the experimental geometry in Fig. 2(c) after population of extreme high-field and low-field seeking states with a $1.0 \mu$ s application of the decelerating field. 10000 point Monte Carlo simulations are also shown as bold lines assuming a beam temperature of $1 \mathrm{~K}$. The inset shows the Stark spectrum at the point of excitation with the two levels $E$ and $F$ indicated.

flight distribution of the molecules arriving at the detector, and as reported in Ref. 19, for neat $\mathrm{H}_{2}$ the deceleration or acceleration was best observed by allowing the Rydberg molecules to fly all the way to the detector. Figure 13 shows the distribution of arrival times for excitation of an extreme lowfield seeking state $(E)$ and an extreme high-field seeking state $(F)$ of the $n=17$ manifold, while applying the dipole field for $1 \mu \mathrm{s}$. The average arrival time is later for the low-field seeking state, implying that these molecules are decelerated. In the $1 \mu$ s deceleration period the molecules should travel 2.6 $\mathrm{mm}$. The field rises by $\approx 700 \mathrm{~V} / \mathrm{cm}$ and the potential energy of the molecule in the low-field seeking state increases by $\approx 13 \mathrm{~cm}^{-1}$ (the molecules are excited $6.5 \mathrm{~mm}$ in front of the dipole center). Initially the mean kinetic energy of the molecules is $570 \mathrm{~cm}^{-1}$ for a velocity of $2600 \mathrm{~ms}^{-1}$, and the deceleration corresponds to a decrease in the kinetic energy of about $2.3 \%$. Conversely the potential energy of the highfield seeking state decreases as the molecule moves towards the detector and so the molecule is accelerated, gaining a calculated $2.3 \%$ of kinetic energy.

Figure 13 shows Monte Carlo trajectory simulations of the time-of-flight distributions for the two different Stark components. These simulations, comprising 10000 trajectories each, agree well with the experimental observations, both in terms of the shift in the mean time of flight and also the width of the distributions (showing that the parallel temperature of $1 \mathrm{~K}$ that we use in the simulations is a good description of the beam).

Figure 14 shows the time-of-flight profiles for 15 of the components of the $n=17$ Stark manifold recorded under the same conditions as for the deceleration experiments shown in Fig. 13. A wider range of time of flight is shown than previously. The gradual shift of the main peak from 108 to $110 \mu$ s reflects the change from acceleration to deceleration as the field dependence of the energy changes from negative to positive, and the gradual trend confirms the validity of the measured difference between the highest and lowest energy 


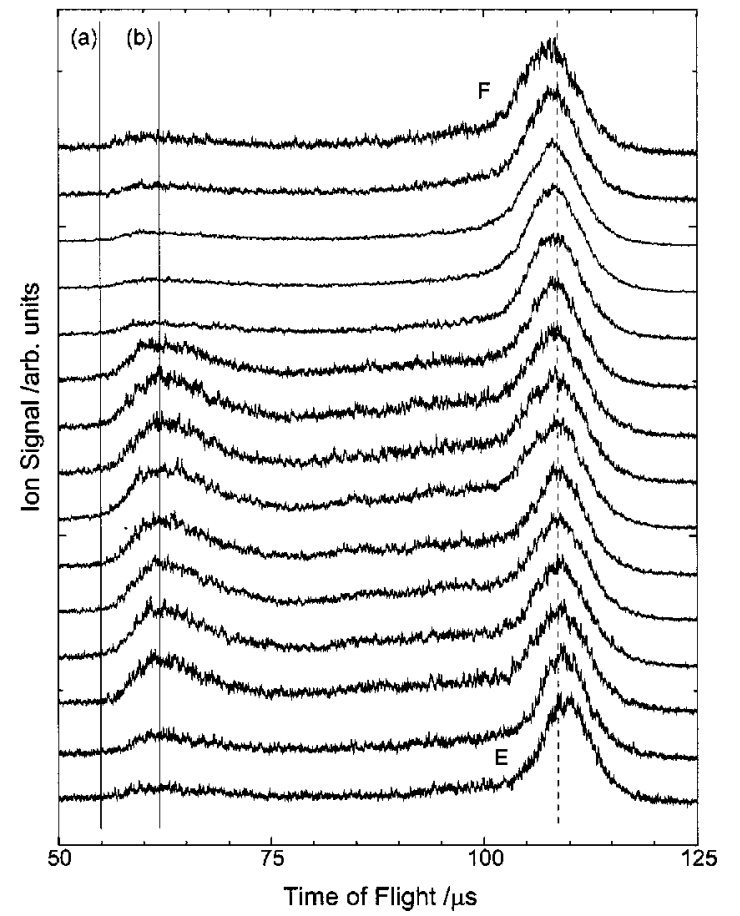

FIG. 14. Time-of-flight profiles for 15 components of the $N^{+}=2, n=17$ Stark manifold after applying the deceleration field for $1.0 \mu$ s (as in Fig. 13). The calculated earliest arrival times for $\mathrm{H}_{2}^{+}$and $\mathrm{H}_{3}^{+}$produced by the collisional mechanisms described in the text are shown as vertical lines labeled (a) and (b), respectively.

components of the manifold. However it is also seen that for all Stark components an earlier peak appears in the time-offlight distribution, at around $62 \mu \mathrm{s}$, most strongly observed for the inner components of the Stark manifold.

Three possible explanations for the earlier component, which must result from decay of the Rydberg states at an early position in the flight tube, are either (a) collisional ionization from room temperature background gases, (b) reactive collisions of $\mathrm{H}_{2}$ Rydberg molecules with $\mathrm{H}_{2}$ ground state molecules in the beam to produce $\mathrm{H}_{3}^{+}$ions, or (c) predissociation to electronically excited fragments. If predissociation is the explanation for this shorter time signal then it must be assumed that electronically excited $\mathrm{H}$ atoms are formed in the process, otherwise field ionization at the detector would not be possible. If the dissociation process yields $\mathrm{H}(n=1)$ $+\mathrm{H}(n=2)$, as shown previously for $n s$ and $n d$ Rydberg series, ${ }^{28,30}$ the $\mathrm{H}$ atoms would acquire approximately 3000 $\mathrm{cm}^{-1}$ of excess energy leading to an axial velocity of 8473 $\mathrm{ms}^{-1}$. If the predissociation occurs in the center of the extraction region the predicted earliest arrival time at the detector for the $\mathrm{H}$ atoms would be only $42 \mu$ s after photoexcitation, compared to the actual onset of $57 \mu$ s and the peak at $62 \mu \mathrm{s}$. On the other hand it is possible that the predissociation does not occur until the molecules pass through the grid at the front of the dynode stack in the time of flight region. The penetrating field from the MCP detector could be sufficient in this region to "unlock" the stability that was conferred on these molecules when the field was switched off earlier. In that case, the predicted earliest time of arrival would be $52 \mu$ s for an $\mathrm{H}$ atom ejected along the flight axis. This time still comes before the onset of the first peak in the

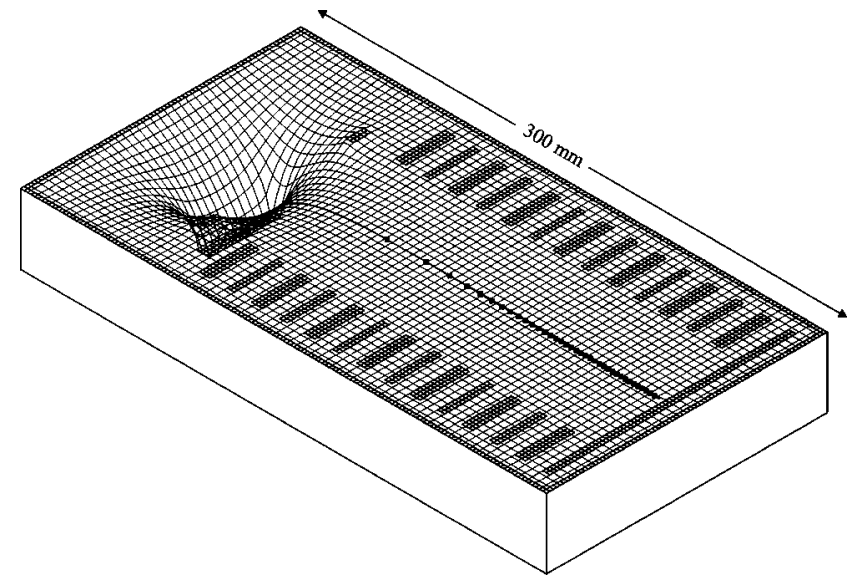

FIG. 15. Simulated fields (Ref. 27) due to penetration of the MCP field into the time-of-flight drift tube of the apparatus. A sample $\mathrm{H}_{2}^{+}$ion trajectory is shown (points at $1 \mu$ s intervals) for an ion entering the drift region at the mean molecular beam velocity $\left(2600 \mathrm{~ms}^{-1}\right)$.

time of flight signal, and it is not easy to provide an explanation as to why a peak should be observed as opposed to a more continuous distribution of $\mathrm{H}$ atoms formed over a range of times throughout the flight tube.

In case (a) or (b), an $\mathrm{H}_{2}{ }^{+}$or an $\mathrm{H}_{3}{ }^{+}$ion might be produced at any point after the grid at the entrance to the dynode stack, and penetrating fields from the MCP would accelerate the ions towards the detector resulting in an earlier arrival time. Figure 15 shows the simulated fields in the time of flight region and a sample $\mathrm{H}_{2}{ }^{+}$ion trajectory with the particle position drawn at $1 \mu$ s intervals. It is evident that the ions only receive significant acceleration after traveling a distance of approximately $7 \mathrm{~cm}$ (about $30 \%$ of the distance into the dynode stack) and until this point they continue to move at approximately at the neutral beam velocity. Consequently all the ions formed in this $7 \mathrm{~cm}$ length region will receive a similar acceleration by the field resulting in a peak in the time of flight distribution. Ions formed later in the time of flight tube will be immediately accelerated and arrive over a spread of later flight times with low intensity. The flight time of $\mathrm{H}_{3}{ }^{+}$and $\mathrm{H}_{2}{ }^{+}$ions would be slightly different, and Fig. 14 indicates with vertical lines the earliest possible arrival time for these ions, based on the time of flight of an ion formed at the entrance grid. This analysis does not allow a clear enough distinction between the two processes. Nevertheless ion formation, either $\mathrm{H}_{2}{ }^{+}$or $\mathrm{H}_{3}{ }^{+}$, should be regarded as a more probable explanation than predissociation based on the time of flight simulations.

If $\mathrm{H}_{2}{ }^{+}$ions are formed through collisions with background gas then this would require the involvement of polar neutral molecules, such as $\mathrm{H}_{2} \mathrm{O}$, as it is known that nonpolar gases are very inefficient at driving $n$-changing processes in Rydberg states ${ }^{34,43,44}$ (ionization can be considered as the limiting case of a large change of $n$ ). The formation of $\mathrm{H}_{3}{ }^{+}$ ions through the reaction of $\mathrm{H}_{2}$ Rydberg states with $\mathrm{H}_{2}$ ground state molecules has been studied in detail ${ }^{45,46}$ and it has been concluded that such reactions occur at a comparable rate to the corresponding ion-molecule reaction $\mathrm{H}_{2}{ }^{+}+\mathrm{H}_{2}$.

A particularly interesting feature of the present results is 
that the inner components of the Stark manifold appear to be more susceptible to the early decay mechanism than the outer components. At the present time no satisfactory explanation can be found for this variation in terms of the collisional processes discussed above, and therefore other mechanisms for producing this early peak cannot be ruled out. The dynamics of the Rydberg states in these experiments will be discussed in more detail in a future paper. ${ }^{42}$

\section{TOWARDS THE TRAPPING OF $\mathrm{H}_{2}$ RYDBERG MOLECULES}

The results presented here demonstrate that nonpolar nonmagnetic molecules can be accelerated or decelerated in an electrostatic field after excitation to Rydberg states. The results were achieved by applying a rather modest field gradient for a time of $1 \mu \mathrm{s}$ or less in a single stage of deceleration or deflection. The observed deceleration, and also the deflection when the dipole is horizontal, agrees well with trajectory calculations. The key questions that follow are, first whether the molecules can be slowed by not just a few percent but actually by $100 \%$ to an average of zero velocity; second whether, even if they were trapped in this way, their lifetimes would be sufficiently long for a useful purpose to be served. If the $\mathrm{H}_{2}$ molecules were seeded in argon then the beam velocity would reduce to $\approx 700 \mathrm{~ms}^{-1}$ and the $\mathrm{H}_{2}$ molecules would have $\approx 40 \mathrm{~cm}^{-1}$ of kinetic energy as opposed to the $570 \mathrm{~cm}^{-1}$ for neat $\mathrm{H}_{2}$. For Rydberg molecules excited to an extreme Stark manifold state at $n=17$, a field rise of 2160 $\mathrm{V} / \mathrm{cm}$ would be required to increase the potential energy by $40 \mathrm{~cm}^{-1}$ and hence remove this amount of kinetic energy. Unfortunately for fields above $1300 \mathrm{~V} / \mathrm{cm}$ complications arise in the Stark map because of overlap of adjacent Stark manifolds of different $n$. Ideally, following the lead shown by Meijer and co-workers in their work on ground-state dipolar molecules, we would arrange for the molecules to pass through a sequence of deceleration stages in which the field rise was limited to less than $1000 \mathrm{~V} / \mathrm{cm}$. However, such an arrangement would be unlikely to work for the Rydberg molecules because the transmission losses, caused by decay and state-changing processes, are likely to be significant over the long time passing through the multistage electrode system. An alternative approach would be to arrange that the field is periodically stepped down as the decelerating molecules approach the dipole so that the field never reaches more than $1000 \mathrm{~V} / \mathrm{cm}$, even though the molecule always experiences an increasing field as it moves towards the dipole.

In any case it is clear that seeding the $\mathrm{H}_{2}$ in a heavier carrier gas would be a useful first step towards full deceleration. Time of flight profiles are shown in Fig. 16 for the case where the $\mathrm{H}_{2}$ was seeded in helium ( $\left.\mathrm{He}: \mathrm{H}_{2}=20: 1\right)$ resulting in a beam that was approximately $33 \%$ slower (mean velocity $\approx 1740 \mathrm{~ms}^{-1}$ ), such that the kinetic energy of the $\mathrm{H}_{2}$ molecules is reduced by a factor of 2.2. A proportion of the Rydberg states are seen to survive the longer transit time to the detector $(165 \mu \mathrm{s})$ although the signal level is significantly lower than for the unseeded beam. If the extreme high-field seeking state of the $n=17$ manifold is populated and the dipole field is applied for $1.3 \mu$ s then a change of $6 \%$ of the

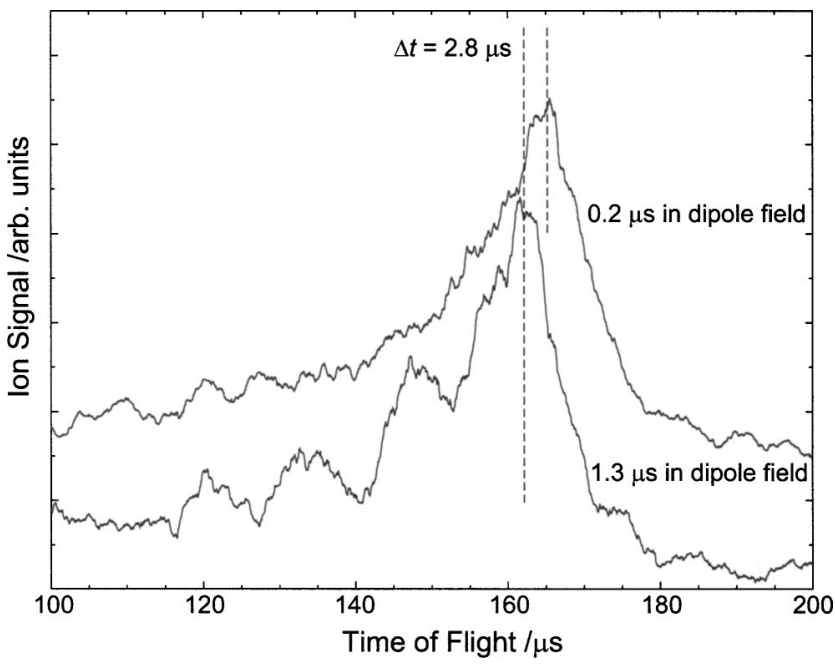

FIG. 16. Time-of-flight profiles for acceleration of the most redshifted Stark state of the $n=17$ manifold (level $E$ ), with the $\mathrm{H}_{2}$ seeded in helium gas $\left(\mathrm{He}: \mathrm{H}_{2}=20: 1\right)$ and the molecules exposed to the dipole field for 1.3 or $0.2 \mu \mathrm{s}$.

initial kinetic energy is achieved (in this case an acceleration).

Seeding in neon has also been attempted but the time of flight to the detector (estimated to be $300 \mu \mathrm{s}$ ) was too long to obtain a measurable Rydberg field ionization signal. Therefore an alternative strategy was adopted in which field ionization of the Rydberg states passing through the dipole was carried out by applying a pulsed field $(3330 \mathrm{~V} / \mathrm{cm})$ across grids 2 and 3 after approximately $12 \mu$ s. It was found that, under these conditions, the changes in the time-of-flight distribution of ions arriving at the detector for different Stark states were too small to measure, because the ions arriving earlier in the ionization region receive less acceleration than those arriving later. However, it was possible to observe an acceleration or deceleration of the Rydberg molecules by measuring the total ion signal as a function of the timing of the field ionization pulse. If the molecules are decelerated then they will arrive later in the field ionization region and a signal will only be observed if the field-ionization/ acceleration pulse is delayed to coincide with the presence of the bulk of the Rydberg molecules in the field ionization region. The effect is shown in Fig. 17 where the onset of the field ionization signal does not occur until a later delay time for population of the extreme low-field seeking state compared to an extreme high-field seeking state of the $n=17$ manifold. For these measurements the kinetic energy of the $\mathrm{H}_{2}$ molecules is reduced or increased by approximately $9 \%$ of its initial value for application of the dipole field for 2.2 $\mu$ s (from an initial kinetic energy of $91 \mathrm{~cm}^{-1}$ ).

With regard to the issue of lifetimes, the demonstration that the Rydberg states survive all the way to the detector $(>100 \mu$ s flight time) is of significance in the context of trapping Rydberg molecules even though this behavior will only be achievable if the field is switched to near zero after a few $\mu$ s. Assuming that the molecules can be slowed to nearzero velocity in the first few $\mu$ s, the fields that would be required to trap them in a harmonic potential would be very low and therefore it is likely that these fields would not be 


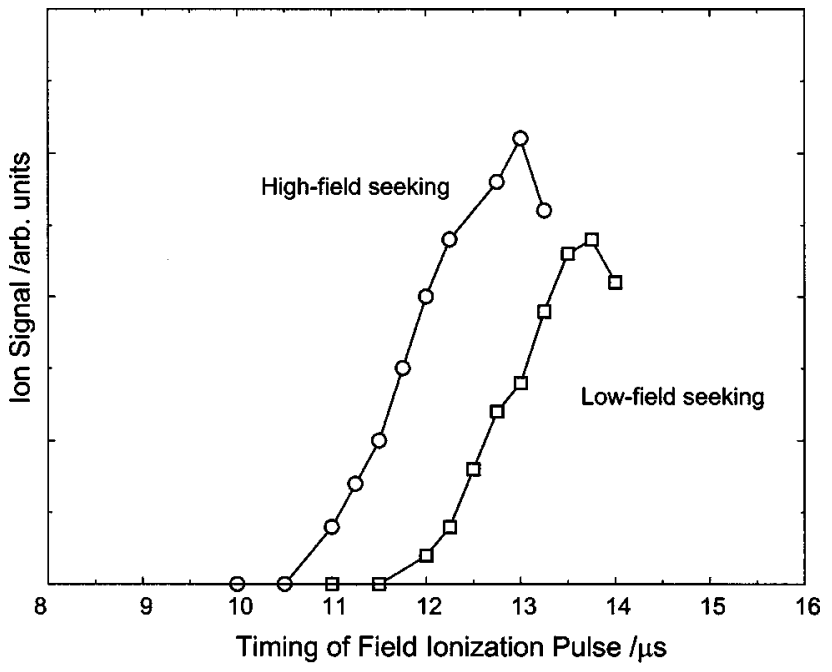

FIG. 17. Field ionization signal as a function of delay of the field ionization pulse between grids 2 and 3 after population of the most redshifted (highfield seeking) and the most blueshifted (low-field seeking) Stark states of the $n=17$ manifold.

large enough to reintroduce decay to these states. There would therefore appear to be genuine possibilities of trapping the Rydberg molecules on a time scale of hundreds of $\mu$ s, offering remarkable opportunities to investigate and exploit the properties of a bunch of cold strongly interacting molecules.

Finally we point out that all neutral molecules possess Rydberg states that are likely to display a very similar Stark effect to that shown here, even though there may be additional complications of spectroscopic selection when the vibration-rotation spacings of the ionic core are small. Thus, this technique is, in principle, quite general, and we suggest here that it has the potential to have an impact on the design of future experiments aimed at studying the properties of ultracold molecules.

\section{ACKNOWLEDGMENTS}

We are grateful to the Ramsay Memorial Trust for a Fellowship to one of us (Y.Y.), to the EPSRC for support of this work, and to the Royal Society for providing financial support to allow research exchange visits between members of the T.P.S. and F.M. groups. F.M. thanks the T.P.S. group for their hospitality during a research visit under this scheme.

${ }^{1}$ Atomic and Molecular Beam Methods, edited by G. Scoles (Oxford University Press, Oxford, 1988), Vol. 1.

${ }^{2}$ S. Chu, Rev. Mod. Phys. 70, 685 (1998).

${ }^{3}$ M. H. Anderson, J. R. Ensher, M. R. Matthews, C. E. Wieman, and E. A. Cornell, Science 269, 168 (1995).

${ }^{4}$ W. D. Phillips, Rev. Mod. Phys. 70, 721 (1998).

${ }^{5}$ H. J. Metcalf and P. van der Straten, Laser Cooling and Trapping (Springer, New York, 1999).
${ }^{6}$ W. C. Stwalley, in Interactions of Cold Atoms and Molecules, edited by P. Soldan, M. T. Cvitas, J. M. Hutson, and C. S. Adams (University of Durham, Press, Durham, 2002), p. 1.

${ }^{7}$ J. M. Doyle, B. Friedrich, J. Kim, and D. Patterson, Phys. Rev. A 52, R2515 (1995).

${ }^{8}$ H. L. Bethlem, G. Berden, and G. Meijer, Phys. Rev. Lett. 83, 1558 (1999).

${ }^{9}$ H. L. Bethlem, F. M. H. Crompvoerts, R. T. Jongma, J. Y. T. van de Meerakker, and G. Meijer, Phys. Rev. A 65, 053416 (2002).

${ }^{10}$ H. L. Bethlem and G. Meijer, Int. Rev. Phys. Chem. 22, 73 (2003).

${ }^{11}$ A. Fioretti, D. Comparat, A. Crubellier, O. Dulieu, F. Masnou-Seeuws, and P. Pillet, Phys. Rev. Lett. 80, 4402 (1998).

${ }^{12}$ R. Wymar, R. S. Freeland, D. J. Han, C. Ryu, and D. J. Heinzen, Science 287, 1016 (2000).

${ }^{13}$ M. W. Zwierlein, C. A. Stan, C. H. Schunck, S. M. F. Raupach, S. Gupta, Z. Hadzibabic, and W. Ketterle, Phys. Rev. Lett. 91, 250401 (2003).

${ }^{14}$ D. H. Parker and R. B. Bernstein, Annu. Rev. Phys. Chem. 40, 561 (1989).

${ }^{15}$ S. E. Novick, P. B. Davies, P. B. Dyke, and W. Klemperer, J. Am. Chem. Soc. 95, 8547 (1973).

${ }^{16} \mathrm{~J}$. Reuss, in Atomic and Molecular Beam Methods, edited by G. Scoles (Oxford University Press, Oxford, 1988), Vol. 1, p. 277.

${ }^{17}$ A. L. Goodgame and T. P. Softley, J. Phys. B 32, 4839 (1999).

${ }^{18}$ D. Townsend, A. L. Goodgame, S. R. Procter, S. R. Mackenzie, and T. P. Softley, J. Phys. B 34, 439 (2001).

${ }^{19}$ S. R. Procter, Y. Yamakita, F. Merkt, and T. P. Softley, Chem. Phys. Lett. 374, 667 (2003).

${ }^{20}$ E. Vliegen, H. J. Wörner, T. P. Softley, and F. Merkt, Phys. Rev. Lett. 92, 033005 (2004).

${ }^{21}$ W. H. Wing, Phys. Rev. Lett. 45, 631 (1980).

${ }^{22}$ T. Breeden and H. Metcalf, Phys. Rev. Lett. 47, 1726 (1981).

${ }^{23}$ A. A. Radzig and B. M. Smirnov, Reference Data on Atoms Molecules and Ions (Springer, New York, 1985), p. 396.

${ }^{24}$ J. M. Gilligan and E. E. Eyler, Phys. Rev. A 46, 3676 (1992).

${ }^{25}$ M. L. Brewer, J. S. Hulmer, and D. E. Manolopoulos, J. Chem. Phys. 106, 4832 (1997).

${ }^{26}$ S. R. Procter, D. Phil. thesis, Oxford University, Oxford, 2004.

${ }^{27}$ D. A. Dahl, SIMION 3D package, version 6.0 (1995), version 7.0 available.

${ }^{28}$ A. Osterwalder, Ph.D. thesis, ETH Zurich, 2002.

${ }^{29}$ W. L. Glab, K. Qin, and M. Bistransin, J. Chem. Phys. 102, 2338 (1995).

${ }^{30} \mathrm{H}$. Rottke and K. H. Welge, J. Chem. Phys. 97, 908 (1992).

${ }^{31}$ H. H. Fielding and T. P. Softley, Chem. Phys. Lett. 185, 199 (1991).

${ }^{32}$ W. E. Ernst, T. P. Softley, and R. N. Zare, Phys. Rev. A 37, 4172 (1988).

${ }^{33}$ A. L. Goodgame, H. Dickinson, S. R. Mackenzie, and T. P. Softley, J. Chem. Phys. 116, 4922 (2002).

${ }^{34}$ T. F. Gallagher, Rydberg Atoms, Cambridge Monographs on Atomic Molecular and Optical Physics No. 3 (Cambridge University Press, Cambridge, 1994).

${ }^{35}$ Ch. Bordas and H. Helm, Phys. Rev. A 45, 387 (1992).

${ }^{36}$ M. J. J. Vrakking, J. Chem. Phys. 105, 7336 (1996).

${ }^{37}$ K. Qin, M. Bistransin, and W. L. Glab, Phys. Rev. A 47, 4154 (1993).

${ }^{38}$ S. C. Ross and Ch. Jungen, Phys. Rev. A 49, 4353 (1994).

${ }^{39}$ R. E. Moss, Mol. Phys. 80, 1541 (1993).

${ }^{40}$ L. Y. Baranov, A. Held, H. L. Selzle, and E. W. Schlag, Chem. Phys. Lett. 291, 311 (1998)

${ }^{41}$ S. R. Procter, M. J. Webb, and T. P. Softley, Faraday Discuss. 115, 277 (2000) and references therein.

${ }^{42}$ S. R. Procter, E. Vliegen, H.-J. Woerner, A. L. Goodgame, F. Merkt, and T. P. Softley (unpublished).

${ }^{43}$ H. Hotop and A. Niehaus, J. Chem. Phys. 47, 2506 (1967).

${ }^{44}$ T. P. Softley, Int. Rev. Phys. Chem. 23, 1 (2004).

${ }^{45}$ S. T. Pratt, J. L. Dehmer, P. M. Dehmer, and W. A. Chupka, J. Chem. Phys. 101, 882 (1994)

${ }^{46}$ P. M. Dehmer and W. A. Chupka, J. Phys. Chem. 99, 1686 (1995). 